

\section{Headgroup Structure and Cation Binding in Phosphatidylserine Lipid Bilayers}

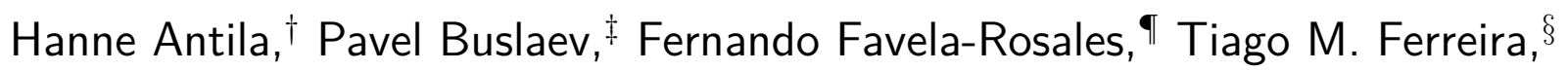
Ivan Gushchin, ${ }^{\ddagger}$ Matti Javanainen, $"$ Batuhan Kav, ${ }^{\dagger}$ Jesper J. Madsen, ${ }^{\perp, \#}$ Josef Melcr, ${ }^{@} @$ Markus S. Miettinen, ${ }^{\dagger}$ Jukka Määttä, ${ }^{\triangle}$ Ricky Nencini, $\|$ O. H. Samuli Ollila, ${ }^{*} \|, \nabla$ and Thomas J. Piggot ${ }^{\dagger \dagger}$

$\dagger$ Department of Theory and Bio-Systems, Max Planck Institute of Colloids and Interfaces, 14424 Potsdam, Germany

$\ddagger$ Moscow Institute of Physics and Technology, Dolgoprudny, Russia

\Departamento de Investigación, Tecnológico Nacional de México, Campus Zacatecas Occidente, México

$\S N M R$ group - Institute for Physics, Martin-Luther University Halle-Wittenberg, Germany |Institute of Organic Chemistry and Biochemistry of the Czech Academy of Sciences,

Flemingovo nám. 542/2, CZ-16610 Prague 6, Czech Republic

$\perp$ Department of Chemistry, The University of Chicago, Chicago, Illinois, United States of America

\#Department of Global Health, College of Public Health, University of South Florida, Tampa, Florida, United States of America

@ Groningen Biomolecular Sciences and Biotechnology Institute and The Zernike Institute for Advanced Materials, University of Groningen, 9747 AG Groningen, The Netherlands

$\triangle$ Department of Chemistry, Aalto University, Espoo, Finland

$\nabla$ Institute of Biotechnology, University of Helsinki, Finland

$\dagger \dagger$ Chemistry, University of Southampton, Highfield, Southampton SO17 1BJ, United Kingdom

E-mail: samuli.ollila@helsinki.fi 


\begin{abstract}
Phosphatidylserine (PS) is a negatively charged lipid type commonly found in eukaryotic membranes, where it interacts with proteins via nonspecific electrostatic interactions as well as via specific binding. Moreover, in the presence of calcium ions, PS lipids can induce membrane fusion and phase separation. Molecular details of these phenomena remain poorly understood, partly because accurate models to interpret the experimental data have not been available. Here we gather a set of previously published experimental NMR data of C-H bond order parameter magnitudes, $\left|S_{\mathrm{CH}}\right|$, for pure PS and mixed PS:PC (phosphatidylcholine) lipid bilayers, and augment this data set by measuring the signs of $S_{\mathrm{CH}}$ in the PS headgroup using S-DROSS solid-state NMR spectroscopy. The augmented data set is then used to assess the accuracy of the PS headgroup structures in, and the cation binding to, PS-containing membranes in the most commonly used classical molecular dynamics (MD) force fields including CHARMM36, Lipid17, MacRog, Slipids, GROMOS-CKP, Berger, and variants. We show large discrepancies between different force fields, and that none of them reproduces the NMR data within experimental accuracy. However, the best MD models can detect the most essential differences between PC and PS headgroup structures. The cation binding affinity is, in line with our previous results for PC lipids, not captured correctly by any of the PS force fields. Moreover, the simulated response of PS headgroup to bound ions can differ from experiments even qualitatively. The collected experimental dataset and simulation results will pave the way for development of lipid force fields that correctly describe the biologically relevant negatively charged membranes and their interactions with ions. This work is part of the NMRlipids open collaboration project (nmrlipids.blogspot.fi).
\end{abstract}

\title{
Introduction
}

Phosphatidylserine (PS) is the most common negatively charged lipid type in eukaryotic biomembranes. In red blood cells, for example, PS lipids compose $8.5 \%$ of the total lipid 
weight. ${ }^{1}$ The abundance, however, varies between different cells and organelles, and up to $25-35 \%$ of the cytosolic leaflet of plasma membranes consists of PS lipids. ${ }^{2,3}$ PS lipids are important biomolecules that interact with signaling proteins, ${ }^{2}$ regulate surface charge and protein localization, ${ }^{4}$ and induce protein aggregation. ${ }^{5,6}$ Some protein domains interact specifically with PS lipids, while other protein sites attract PS lipids by nonspecific electrostatics and the binding can be regulated by calcium. ${ }^{2}$ Therefore, deciphering the structural details of lipid headgroups and the details of cation binding is crucial for understanding PS-mediated processes on cellular membranes.

Experimental studies have indicated that the serine-containing headgroup of PS is more rigid than the choline-containing headgroup of PC (phosphatidylcholine), owing possibly to electrostatic interactions or formation of a hydrogen bond network between the headgroups. ${ }^{7,8}$ While most monovalent ions interact weakly with PS-containing bilayers, multivalent cations and $\mathrm{Li}^{+}$are able to form strong dehydrated molecular complexes with PS lipids. ${ }^{9-19}$ The dehydrated complexes of PS headgroups with calcium ions can even lead to phase separation. ${ }^{9,10,14-18}$ Mixing PS lipids with PC lipids reduces the propensity of PS to form complexes with multivalent ions and makes the PS headgroup less rigid. ${ }^{7,8,17,18}$ That being said, some studies suggest that $\mathrm{Ca}^{2+}$ has similar specific binding affinity to both negatively charged and zwitterionic phospholipids, and that the increased cation binding to PS lipid bilayers is non-specific and arises only due to the increased local cation concentration in the vicinity of the membrane..$^{20,21}$

Molecular level interpretations of the rigidity of PS headgroup and its interactions with ions are currently lacking. Classical molecular dynamics (MD) simulations have been widely used in efforts to understand the PS headgroup structure, its influence on lipid bilayer properties, and PS interaction with ions. ${ }^{19,22-34}$ Unfortunately, the results have depended on the particular force field used. For example, recent simulations using the NBfix parameters for calcium ${ }^{35}$ in the CHARMM36 force field ${ }^{29,36}$ combined with 2D infrared spectroscopy, suggest that $\mathrm{Ca}^{2+}$ ions interact only with the carboxylate group of PS lipids. ${ }^{33}$ In contrast, 
results from the same lipid model but without the NBfix ion parameters, combined with NMR chemical shifts and rotational-echo double-resonance (REDOR) experiments, indicate a significant binding affinity also toward the phosphate region. ${ }^{34}$ Meanwhile, simulations with the Berger force field, ${ }^{24,37}$ combined with fluorescent and vibrational sum frequency spectroscopy, suggest substantial calcium binding to the carbonyls in the acyl chains. ${ }^{32}$

We have recently demonstrated that the lipid C-H bond order parameters, $S_{\mathrm{CH}}$, can be used to resolve such controversies. ${ }^{38,39}$ The $S_{\mathrm{CH}}$ can be measured from NMR experiments with high accuracy and directly compared to MD simulations to evaluate the quality of the force field and to interpret the experiments. ${ }^{40}$ Using this approach, it has been established that the structure of PC lipid headgroup and glycerol backbone are not well captured by most MD force fields, ${ }^{38}$ that cation binding to PC lipid bilayers is overestimated, ${ }^{39}$ and that the inequivalence of the order parameters of the distinct $\mathrm{C}-\mathrm{H}$ bonds at the carbon 2 in $s n-2$ lipid tail is correctly reproduced only in the CHARMM36 force field. ${ }^{41}$

Here, we first extend the available set of experimentally measured PS lipid headgroup and glycerol backbone $\mathrm{C}-\mathrm{H}$ bond order parameters by measuring the signs of these order parameters using S-DROSS solid-state NMR spectroscopy. Based on the collected experimental data, we then assess the quality of headgroup structures and the ion binding affinity in the available MD simulation models of PS lipids. Our results pave the way for development of lipid models that correctly describe the headgroup region of negatively charged lipids under physiological salt conditions. Such force fields are expected to be extremely useful in understanding the biological functions of lipid headgroups and glycerol backbone, as these are known to behave similarly in simple model membranes and in cells. ${ }^{20,42,43}$ 


\section{Methods}

\section{Experimental $\mathbf{C}-\mathbf{H}$ bond order parameters}

The magnitudes of headgroup and glycerol backbone $\mathrm{C}-\mathrm{H}$ bond order parameters of 1palmitoyl-2-oleoyl-sn-glycero-3-phospho-L-serine (POPS) were determined by measuring the chemical-shift-resolved dipolar splittings with a R-type Proton Detected Local Field (R-PDLF) experiment. ${ }^{44}$ The corresponding order parameter signs were measured with a S-DROSS experiment ${ }^{45}$ using natural abundance ${ }^{13} \mathrm{C}$ solid state NMR spectroscopy as described previously. ${ }^{46,47}$ The experiments were done in a Bruker Avance III 400 spectrometer operating at a ${ }^{1} \mathrm{H}$ Larmor frequency of $400.03 \mathrm{MHz}$. Magic angle spinning (MAS) of the sample was used at a frequency of $5.15 \mathrm{kHz}$ (R-PDLF) and $5 \mathrm{kHz}$ (S-DROSS). The following experimental setups were used.

$C-H$ bond order parameters from the $R-P D L F$ experiment. The parameters are described according to Figures $1 \mathrm{c}$ and $2 \mathrm{c}$ of the original reference for the R-PDLF experiment. ${ }^{44}$ The refocused-INEPT delays were $\tau_{1}=1.94 \mathrm{~ms}$ and $\tau_{2}=0.97 \mathrm{~ms}$. The used radio frequency pulses had the following nutation frequencies: $46.35 \mathrm{kHz}$ (R18 ${ }_{1}^{7}$ pulses), $63.45 \mathrm{kHz}\left({ }^{13} \mathrm{C} 90^{\circ}\right.$ and $180^{\circ}$ ), $50 \mathrm{kHz}$ (SPINAL64 ${ }^{1} \mathrm{H}$ decoupling pulses). The $t_{1}$ increment was equal to $10.79 \mu \mathrm{s}$ $\times 18 \times 2$, and 32 points in the indirect dimension were recorded using 1024 scans for each, with a recycle delay of $5 \mathrm{~s}$ and a spectral width of $149.5 \mathrm{ppm}$.

Order parameter signs from the S-DROSS experiment. The parameters are described according to Figures $1 \mathrm{~b}$ and $1 \mathrm{c}$ of the original reference for the S-DROSS experiment. ${ }^{45}$ The refocused-INEPT delay $\delta_{2}$ was $1.19 \mathrm{~ms}$. The $\tau_{1}$ and $\tau_{2}$ in the S-DROSS recoupling blocks $R$ were set as $\tau_{1}=39.4 \mu \mathrm{s}$ and $\tau_{2}=89.4 \mu \mathrm{s}$. The used radio frequency pulses had the nutation frequencies: $63.45 \mathrm{kHz}\left({ }^{13} \mathrm{C} 90^{\circ}\right.$ and $\left.180^{\circ}\right), 50 \mathrm{kHz}\left({ }^{1} \mathrm{H}\right.$ SPINAL64 decoupling). The $t_{1}$ increment (dipolar recoupling dimension) was $800 \mu \mathrm{s}$, and a total of 8 points along $t_{1}$ were measured using 1024 scans for each, with a recycle delay of $5 \mathrm{~s}$ and a spectral width of $149.5 \mathrm{ppm}$. 
Numerical simulations of S-DROSS curves. The numerical simulations of S-DROSS curves were performed using the SIMPSON simulation package ${ }^{48}$ by inputing the ${ }^{13} \mathrm{C}-{ }^{1} \mathrm{H}$ dipolar couplings, either as determined by the R-PDLF experiments, or as calculated from the known ${ }^{2} \mathrm{H}$ quadrupolar couplings. ${ }^{7}$ The chemical shift anisotropy and homonuclear couplings were neglected, and the SIMPSON input file rep2000 was used to simulate a random distribution of bilayer orientations in the samples studied.

Sample preparation. The sample was prepared simply by mixing POPS powder (1palmitoyl-2-oleoyl-sn-glycero-3-phospho-L-serine, purchased from Avanti Polar Lipids as sodium salt) with water (lipid:water 60:40 wt-\%) in an Eppendorf tube, centrifuging the mixture and stirring with a thin glass rod repeatedly (approximately 5 to 6 times centrifuging/stirring) until a homogeneous viscous fluid was visually observed. Then $20 \mathrm{mg}$ of the sample was transferred to an NMR insert suitable for $4 \mathrm{~mm}$ NMR rotors.

\section{Molecular dynamics simulations}

Molecular dynamics simulation data were collected using the Open Collaboration method, ${ }^{38}$ with the NMRlipids Project blog (nmrlipids.blogspot.fi) and GitHub repository (github. com/NMRlipids/NMRlipidsIVotherHGs) as the communication platforms. The simulated systems are listed in Tables 1 (pure PS bilayers without additional ions) and 2 (mixed PC:PS bilayers at various salt concentrations). Further simulation details are given in the SI, and the simulation data are indexed in a searchable database available at www.nmrlipids.fi, and in the NMRlipids/MATCH repository (github.com/NMRlipids/MATCH). ${ }^{49}$

The $\mathrm{C}-\mathrm{H}$ bond order parameters were calculated directly from the carbon and hydrogen positions using the definition

$$
S_{\mathrm{CH}}=\frac{1}{2}\left\langle 3 \cos ^{2} \theta-1\right\rangle,
$$

where $\theta$ is the angle between the $\mathrm{C}-\mathrm{H}$ bond and the membrane normal (taken to align with $z$, with membrane in the $x y$-plane). Angular brackets denote average over all sampled 


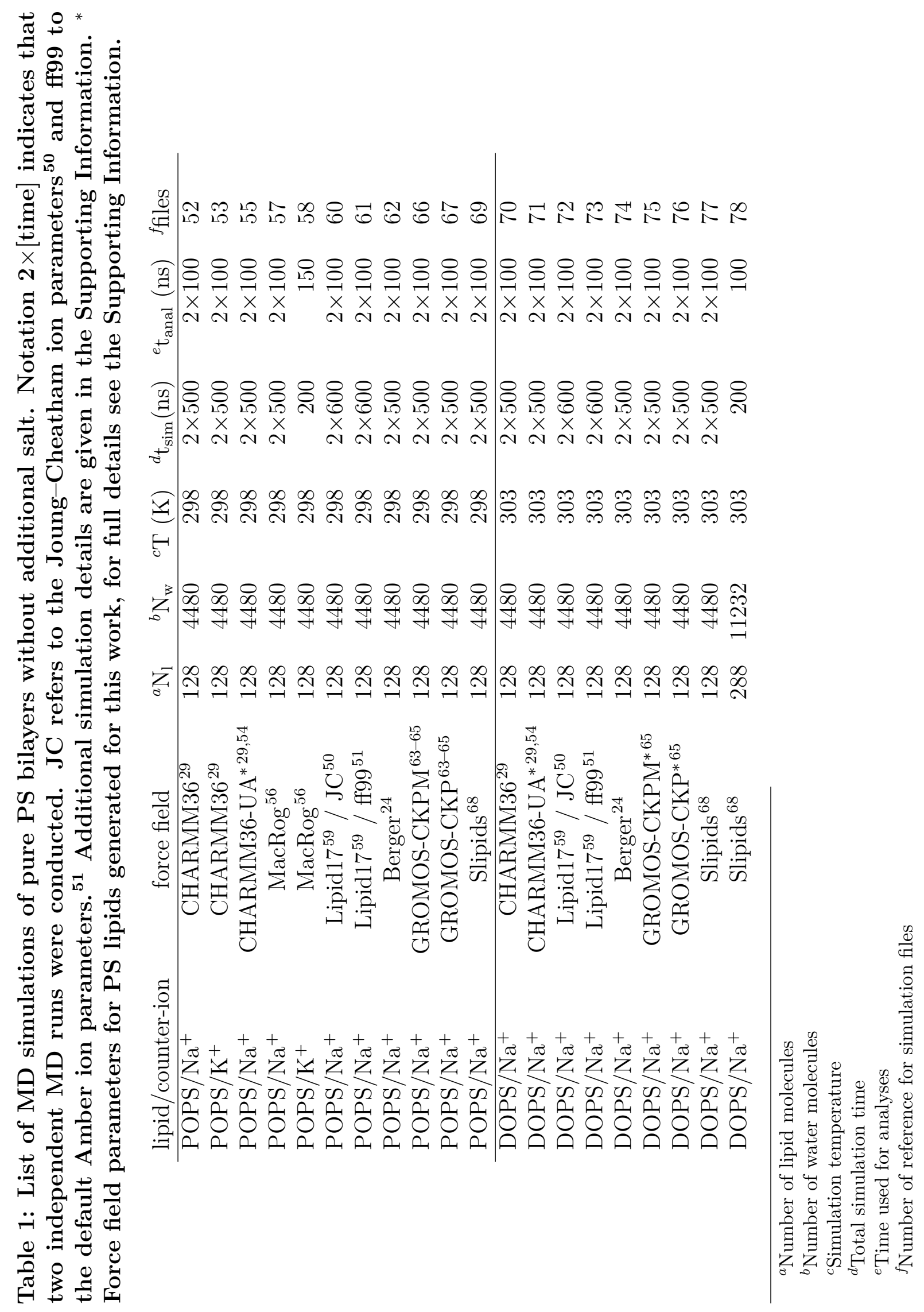




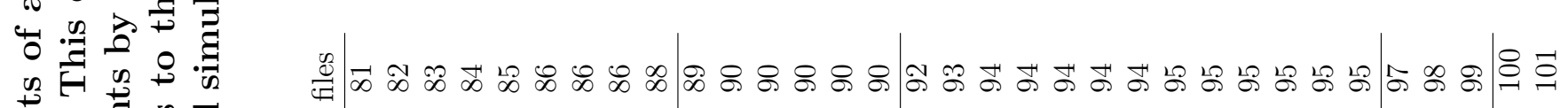

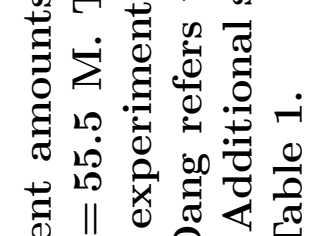

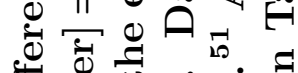

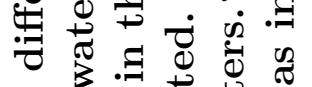

E

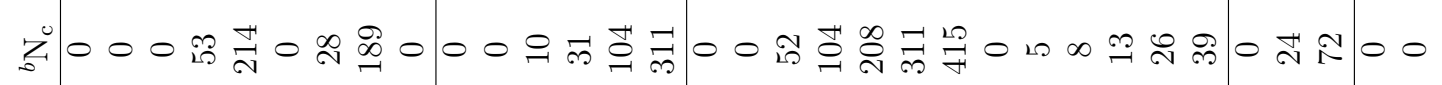

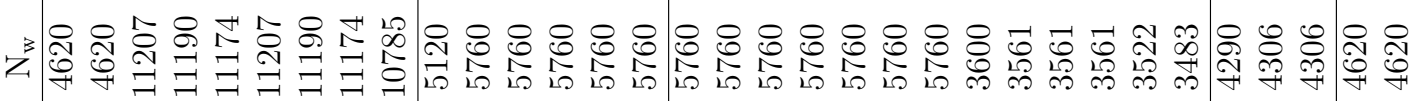
ปก

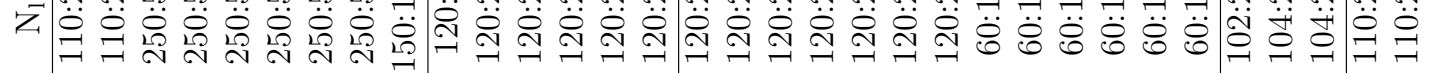

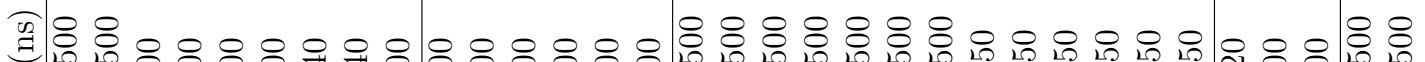

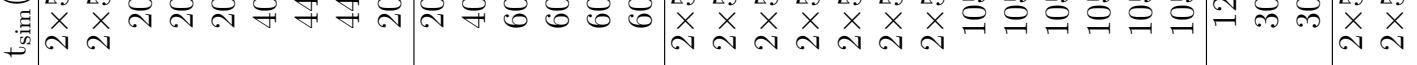

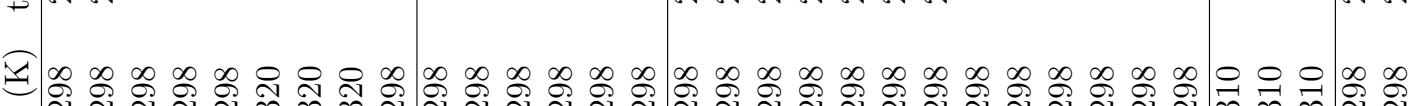

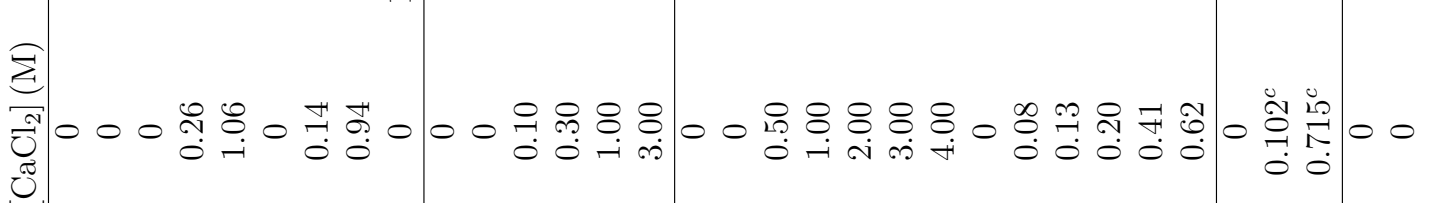

๘

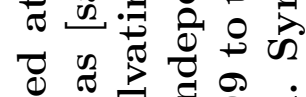

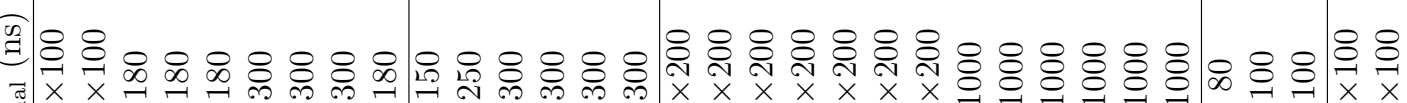

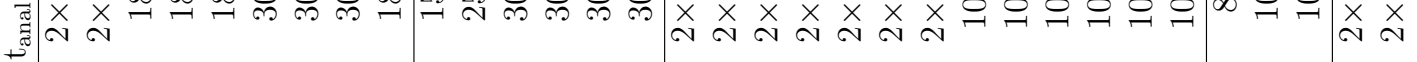


configurations. The order parameters were calculated by first averaging over time separately for each lipid in the system, and then calculating the average and the standard error of the mean over the different lipids. The analysis can be conducted using the Python program calcOrderParameters.py, available in Ref. 49, that uses the MDAnalysis library. ${ }^{102,103}$ For the united atom force fields, the positions of hydrogens were generated before the order parameter calculation using the protonate tool of the Gromacs 3 sofware package. ${ }^{104}$ The ion number density profiles were calculated using the gmx density tool of the Gromacs sofware package. ${ }^{104}$

\section{Using the molecular electrometer to compare ion binding to nega- tively charged lipid bilayers in simulations and in experiments}

The $S_{\mathrm{CH}}$ of the $\alpha$ and $\beta$ carbons in the PC headgroup decrease proportionally to the amount of positive charge bound to the bilayer, ${ }^{105-107}$ and can therefore be used to measure the ion binding affinity. In addition to ions, the correlation between bound charge and headgroup order parameter change is empirically observed also for peptides, charged amphiphiles, local anesthetics and charged lipids. ${ }^{43,108}$ This concept, known as the molecular electrometer, is especially useful for comparison between simulations and experiments, as the headgroup $S_{\mathrm{CH}}$ at varying cation concentrations can be easily calculated from simulations. ${ }^{39}$ The headgroup $S_{\mathrm{CH}}$ of negatively charged PS and PG lipids also exhibit systematic dependencies on the bound charge, but these are less well understood than for PC. ${ }^{17,109-111}$ Therefore, measuring the PC headgroup $S_{\mathrm{CH}}$ in mixed (here PS:PC) bilayers ${ }^{17,18,111}$ (see also Supporting Information section S2) provides a more straightforward way of characterizing ion binding to such negatively charged membranes.

Calibrating the PC headgroup $S_{\mathrm{CH}}$ response to a known amount of bound charge ${ }^{39,112}$ is an important preliminary step for using the molecular electrometer. This can be done using experimental data from mixtures of PC and monovalent cationic surfactants (such as POPC and dihexadecyldimethylammonium, see SI section S3). ${ }^{112,113}$ Additionally, the response 
of PC headgroup $S_{\mathrm{CH}}$ to the negatively charged PS follows the molecular electrometer in experiments, ${ }^{43}$ which we also quantify here (see SI section S2).

Studies applying the molecular electrometer have used two different definitions for salt

concentration: The concentrations reported either before, ${ }^{17,39,105}$ or after ${ }^{106,112}$ solvating the lipids. In the former case, binding of ions to the lipids leads to a lower bulk concentration than what was present in the original solvent. However, the choice of definition has only a marginal effect to the results in simulations with realistic ion binding affinity (see SI section S4). In this work, we use the concentration before solvating the lipids to be consistent with the reference experimental data. ${ }^{17}$

\section{Results and discussion}

\section{Headgroup and glycerol backbone $\mathrm{C}-\mathrm{H}$ bond order parameters of POPS from ${ }^{13} \mathrm{C}$ NMR}

The INEPT and 2D R-PDLF experiments from POPS samples gave well resolved spectra for all the carbons in the headgroup and glycerol backbone regions (Fig. 1). The glycerol backbone carbon peaks were assigned according to the POPC spectra, ${ }^{46}$ whereas the peaks for $\beta$ and $\alpha$ carbons were assigned according to the $\mathrm{C}-\mathrm{H}$ bond order parameters known from previous ${ }^{2} \mathrm{H}$ NMR experiments. ${ }^{7}$ Slices of the R-PDLF spectra and the resulting $S_{\mathrm{CH}}$ values are shown in the Supporting Information (Fig. S6).

Since the R-PDLF and previous ${ }^{2} \mathrm{H}$ NMR experiments ${ }^{7,18}$ give only the absolute values of $S_{\mathrm{CH}}$, we determined the signs of the PS headgroup $S_{\mathrm{CH}}$ using the S-DROSS experiment. ${ }^{45}$ For a given carbon, its S-DROSS dipolar modulation profile in the indirect dimension is a superposition of sinusoidal functions from the possible orientations of crystallites in the sample (or bilayer patches). We phase corrected the $2 \mathrm{D}$ spectrum in the direct dimension such that positive and negative signs for the $S_{\mathrm{CH}}$ give rise to profiles that initially increase and decrease, respectively. In practice, we use the known negative sign of the acyl chain 

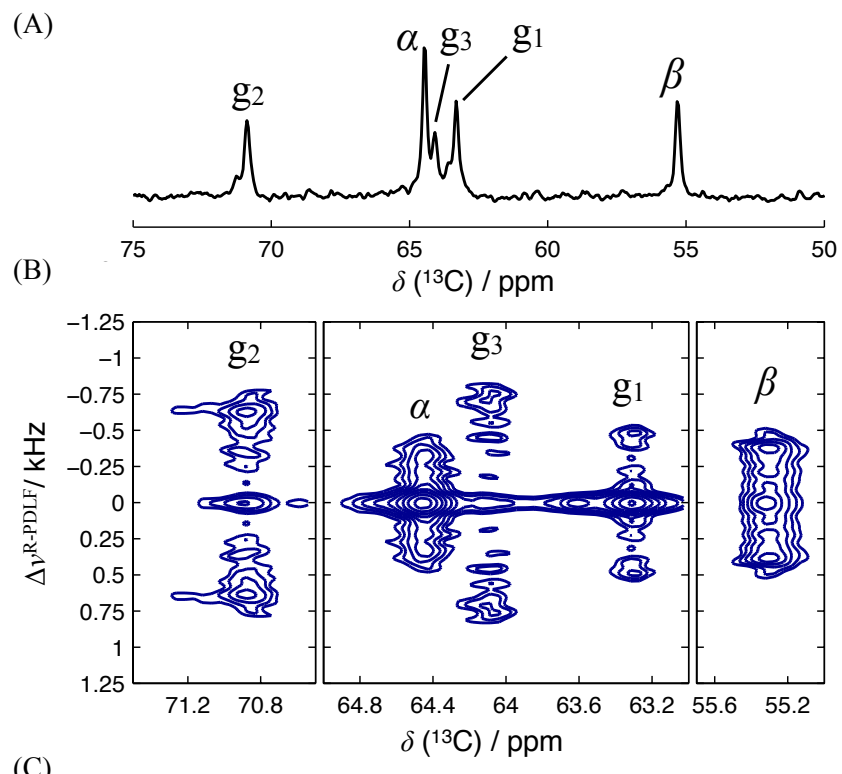

(C)

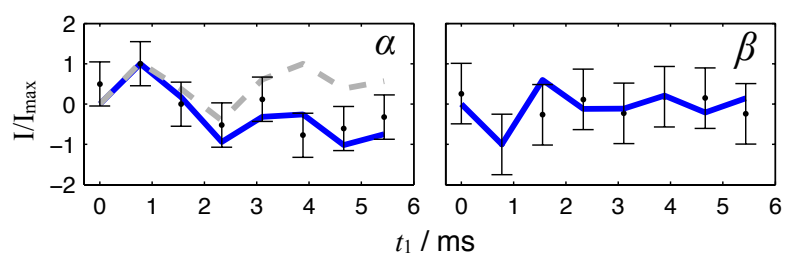

Figure 1: The headgroup and glycerol backbone region of the (A) Refocused-INEPT spectrum and (B) 2D R-PDLF spectra. (C) Experimental S-DROSS data (points), and SIMPSON simulations with the $\mathrm{C}-\mathrm{H}$ bond order parameter values of -0.12 for the $\beta$-carbon and +0.09 and -0.02 for the $\alpha$-carbon (blue lines). Dashed gray line is the S-DROSS curve from a SIMPSON simulation with a positive value $(+0.02)$ for the smaller $\alpha$-carbon $\mathrm{C}-\mathrm{H}$ bond order parameter.

carbons as a reference to perform the phase correction and interpret the distinct initial slopes of the S-DROSS profiles (Fig. S6). The S-DROSS slice for the $\beta$-carbon clearly shows an initial decrease and therefore its order parameter must be negative. For the $\alpha$-carbon such analysis is not as trivial due to the two inequivalent order parameters of the two distinct $\mathrm{C}-\mathrm{H}$ bonds. However, the beginning of its S-DROSS slice suggests that the larger $S_{\mathrm{CH}}$ of the $\alpha$-carbon is positive and the decrease towards negative values at longer $t_{1}$ suggests that the smaller $S_{\mathrm{CH}}$ is negative. This is confirmed by a SIMPSON simulation using the $S_{\mathrm{CH}}$ values of +0.09 from the dipolar coupling measured here (Fig. S6) and -0.02 from the previous ${ }^{2} \mathrm{H}$ NMR experiment. ${ }^{18}$ We used the literature value for the smaller $S_{\mathrm{CH}}$, because the resolution of our R-PDLF experiment was not sufficient to determine the magnitude of 


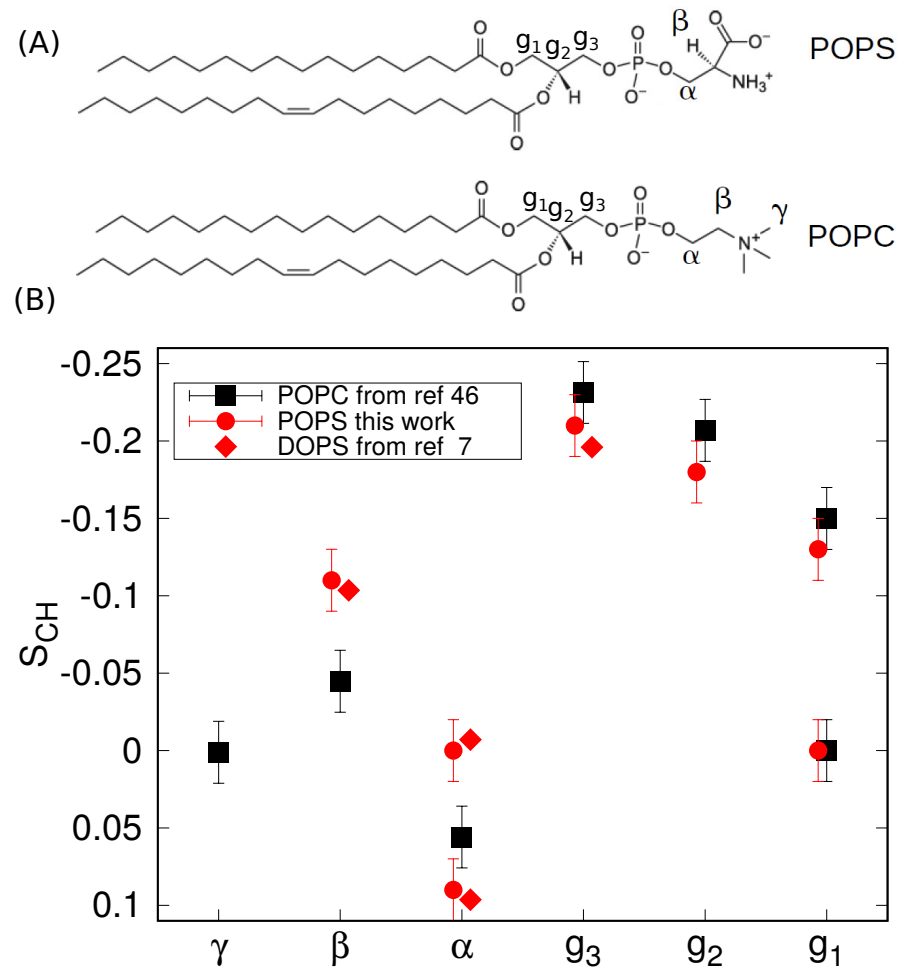

Figure 2: (A) Chemical structures and labels for the headgroup and glycerol backbone carbons. (B) Headgroup and glycerol backbone order parameters of POPS $(\mathrm{T}=298 \mathrm{~K})$ measured in this work compared with the previously published values from DOPS $(T=303 \mathrm{~K}$, ${ }^{2} \mathrm{H}$ NMR, $0.1 \mathrm{M}$ of $\left.\mathrm{NaCl}\right)^{7}$ and POPC $\left(T=300 \mathrm{~K},{ }^{13} \mathrm{C} \mathrm{NMR}\right){ }^{46}$ experiments. Signs of the PS order parameters are measured in this work, whereas signs of the PC order parameters are measured previously. ${ }^{47}$ The size of errorbars $( \pm 0.02)$ shown for ${ }^{13} \mathrm{C} N M R$ data is justified previously. ${ }^{38,40}$

the small value. The S-DROSS curve from a SIMPSON simulation with a positive value for the smaller $S_{\mathrm{CH}}$ (dashed grey in Fig. $1(\mathrm{C})$ ) did not agree with the experiment, corroborating the interpretation that the smaller $S_{\mathrm{CH}}$ is indeed negative.

The headgroup and glycerol backbone order parameters of POPS measured in this work are in good agreement with the previously reported values from ${ }^{2} \mathrm{H} N \mathrm{NR}$ experiments of $\operatorname{DOPS}^{7}$ (Fig. 2). When compared with the previously measured values for POPC ${ }^{46}$ (Fig. 2), the $\beta$-carbon $S_{\mathrm{CH}}$ is significantly more negative, and the $\alpha$-carbon experiences a substantial forking (different $S_{\mathrm{CH}}$ for the two hydrogens in the same carbon ${ }^{40}$ ) in the PS headgroup. These features have been intepreted to arise from a rigid PS headgroup conformation, stabilized by hydrogen bonds or electrostatic interactions, ${ }^{7,8}$ but a detailed structrural interpretation is 
not available.

We note that the DOPS ${ }^{2} \mathrm{H}$ NMR reference data found in the literature ${ }^{7,17}$ was obtained by first solvating the lipids with a buffer solution and then centrifuging the sample to a pellet that was used for the measurements. Such samples have a lower lipid concentration (approximately $10 \mathrm{wt} \%$ of lipids ${ }^{7,17,114}$ ) than the gravimetric samples (60 wt \%) and simulations (approximately 50-60 wt \%) in this work. Larger multilamellar repeat distances are expected in the samples with lower lipid concentrations due to the swelling caused by electrostatic repulsion in pure PS lipid systems. ${ }^{115}$ Yet the PS headgroup $S_{\mathrm{CH}}$ measured from gravimetric samples (POPS) in this work are in good agreement with the results from centrifuged samples. ${ }^{7}$ This, together with the rapid decrease of equilibrium repeat distance with addition of monovalent salt, ${ }^{115,116}$ indicates that the hydration levels of multilamellae are sufficiently similar in the reference experiments and our simulations.

\section{Headgroup and glycerol backbone in simulations of PS lipid bilayers without additional ions}

The different PS MD models produced highly varied headgroup and glycerol backbone $S_{\mathrm{CH}}$ (Fig. 3) and structures (Fig. S9 and section S6 in the Supporting Information). As was previously observed for PC lipids, ${ }^{38}$ also none of the PS models produced a set of $S_{\mathrm{CH}}$ in full quantitative agreement with the experiments. In fact, the models perform less well for PS than for PC (Figs. 3 and 4 vs. Figs. 2 and 4 in Ref. 38), which complicates the interpretation of structural differences between the PC and PS. However, concentrating on the headgroup alone, we see that the best performing models (Slipids, CHARMM36, and CHARMM36-UA) indeed do qualitatively replicate the larger-than-in-PC forking of the $\alpha$-carbon (these models give forkings of $\sim 0.01$ or smaller for $\mathrm{PC}^{38}$ and between $\sim 0.05$ and $\sim 0.12$ for PS) that is observed in experiments (Fig. 2). Additionally, the Slipids force field correctly produces the significantly smaller $\beta$-carbon order parameter for PS $\left(S_{\mathrm{CH}}^{\beta} \approx-0.09\right.$, see Fig. 3$)$ compared to $\mathrm{PC}\left(S_{\mathrm{CH}}^{\beta} \approx-0.03\right.$, see Fig. 2 in Ref. 38), in line with experiments (Fig. 2). 


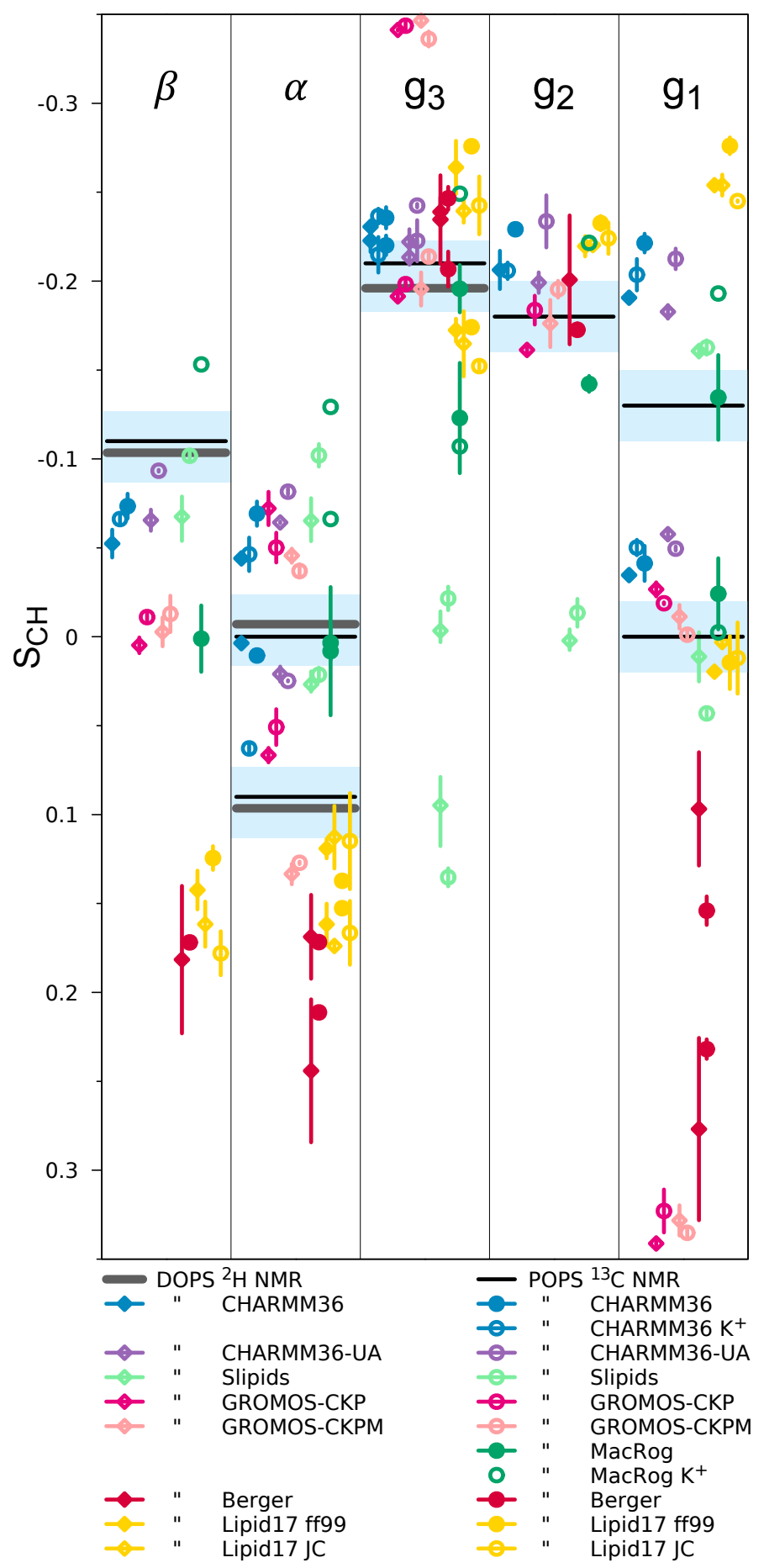

Figure 3: $\mathrm{C}-\mathrm{H}$ bond order parameters, $S_{\mathrm{CH}}$, of the PS headgroup $(\beta$ and $\alpha)$ and glycerol backbone $\left(g_{3}, g_{2}, g_{1}\right)$ carbons from NMR experiments (horizontal lines), and MD simulations with different force fields (symbols). Experimental data for DOPS had been measured with $0.1 \mathrm{M}$ of $\mathrm{NaCl},{ }^{7}$ while all the other data are with counterions only. The data for DOPS is at $303 \mathrm{~K}$ and for POPS at $298 \mathrm{~K}$. The light blue areas span 0.04 units around the average of the extremal experimental values, in accordance with the expected quantitative accuracy of experiments. ${ }^{40}$ The vertical bars shown for all simulation values (excl. MacRog $\mathrm{K}^{+}$) are not error bars, but demonstrate that for these systems we had at least two data sets; the ends of the bars mark the extreme valups from the sets, and the symbol marks their measurement-time-weighted average. 


\begin{tabular}{|c|c|c|c|c|c|c|}
\hline & $\beta$ & $\alpha$ & $g_{3}$ & $g_{2}$ & $g_{1}$ & $\Sigma$ \\
\hline $\begin{array}{c}\text { CHARMM } \\
36 \mathrm{~K}^{+}\end{array}$ & M & M & $\begin{array}{l}M \\
F\end{array}$ & M & $\underset{F}{\mathbf{M}}$ & 7 \\
\hline $\begin{array}{c}\text { CHARMM } \\
36\end{array}$ & M & $\begin{array}{c}\mathbf{M} \\
\mathrm{F}\end{array}$ & M & M & $\begin{array}{c}\text { M } \\
\mathrm{F}\end{array}$ & 8 \\
\hline $\begin{array}{l}\text { CHARMM } \\
\text { 36-UA }\end{array}$ & M & $\mathbf{M}$ & M & $M$ & $\underset{F}{\mathbf{M}}$ & 9 \\
\hline$\underset{\mathrm{K}^{+}}{\text {MacRog }}$ & M & $\underset{F}{\mathbf{M}}$ & $\begin{array}{l}\mathbf{M} \\
\mathbf{F}\end{array}$ & M & $\begin{array}{l}\mathrm{M} \\
\mathbf{F}\end{array}$ & 11 \\
\hline MacRog & $\mathbf{M}$ & $\begin{array}{l}\mathbf{M} \\
\mathbf{F}\end{array}$ & $\begin{array}{c}\mathbf{M} \\
\mathbf{F}\end{array}$ & M & M & 14 \\
\hline $\begin{array}{l}\text { GROMOS- } \\
\text { CKP }\end{array}$ & $\mathbf{M}$ & $\begin{array}{c}M \\
F\end{array}$ & $\begin{array}{c}\mathbf{M} \\
\mathbf{F}\end{array}$ & & $\begin{array}{c}\text { M } \\
F\end{array}$ & 14 \\
\hline $\begin{array}{l}\text { GROMOS- } \\
\text { CKPM }\end{array}$ & $\mathbf{M}$ & $\begin{array}{l}\mathrm{M} \\
\mathbf{F}\end{array}$ & $\begin{array}{c}\mathbf{M} \\
\mathbf{F}\end{array}$ & & $\begin{array}{c}\mathbf{M} \\
\mathbf{F}\end{array}$ & 14 \\
\hline Berger & $\mathbf{M}$ & $\begin{array}{c}\text { M } \\
\mathbf{F}\end{array}$ & $\begin{array}{c}M \\
F\end{array}$ & & $\underset{F}{M}$ & 14 \\
\hline Slipids & M & $\mathbf{M}$ & $\begin{array}{c}\text { M } \\
\text { F }\end{array}$ & $\mathbf{M}$ & $\stackrel{\mathrm{M}}{\mathbf{F}}$ & 14 \\
\hline $\begin{array}{l}\text { Lipid17 } \\
\text { ff99 }\end{array}$ & $\mathbf{M}$ & $\begin{array}{c}\text { M } \\
\mathbf{F}\end{array}$ & $\begin{array}{l}\mathbf{M} \\
\mathbf{F}\end{array}$ & M & $\begin{array}{l}\text { M } \\
F\end{array}$ & 18 \\
\hline $\begin{array}{l}\text { Lipid17 } \\
\text { JC }\end{array}$ & $\mathbf{M}$ & $\underset{\mathrm{F}}{\mathbf{M}}$ & $\begin{array}{l}\mathrm{M} \\
\mathbf{F}\end{array}$ & M & $\begin{array}{l}\mathbf{M} \\
\mathbf{F}\end{array}$ & 18 \\
\hline
\end{tabular}

Figure 4: Rough subjective ranking of force fields based on how far the simulated order parameters were from the experimental values ( $\mathrm{M}$ indicates a magnitude problem) and how well the difference between order parameters of two hydrogens attached to the same carbon were reproduced ( $\mathrm{F}$ indicates a forking problem) in Fig. 3. The letter size increases with problem severity. Color scheme: "within experimental error" (dark green), "almost within experimental error" (light green), "clear deviation from experiments" (light red), and "major deviation from experiments" (dark red). The $\Sigma$-column shows the total deviation of the force field, when individual carbons are given weights of 0 (matches experiment), 1, 2, and 4 (major deviation). For full details of the assessment, see section S10 in the Supporting Information. 


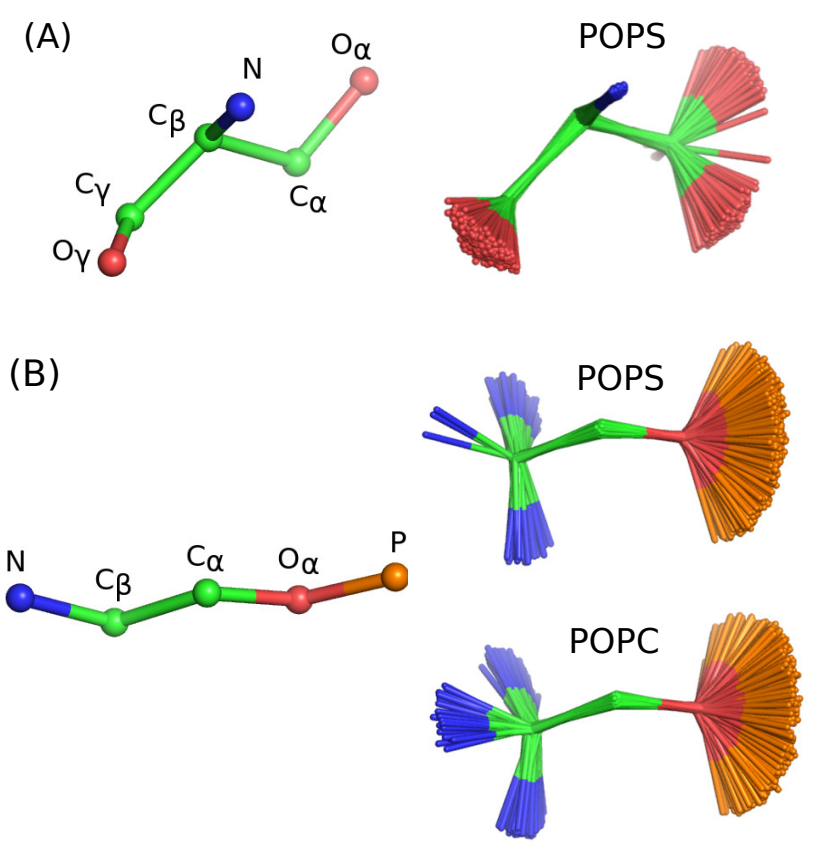

Figure 5: Overlayed snapshots from simulations conducted with CHARMM36 - the force field producing the best agreement with experiments - demonstrate the conformational fluctuations in lipid headgroups. (A) Overlaying the $\mathrm{C}_{\gamma}, \mathrm{C}_{\beta}$, and $\mathrm{C}_{\alpha}$ carbons demonstrates fluctuations around the $\mathrm{O}_{\gamma}-\mathrm{C}_{\gamma}-\mathrm{C}_{\beta}-\mathrm{C}_{\alpha}, \mathrm{C}_{\gamma}-\mathrm{C}_{\beta}-\mathrm{C}_{\alpha}-\mathrm{O}_{\alpha}$, and $\mathrm{N}^{-} \mathrm{C}_{\beta}-\mathrm{C}_{\alpha}-\mathrm{O}_{\alpha}$ dihedrals of the PS headgroup. (B) Overlaying the $\mathrm{C}_{\beta}, \mathrm{C}_{\alpha}$, and $\mathrm{O}_{\alpha}$ atoms demonstrates fluctuations around the $\mathrm{N}-\mathrm{C}_{\beta}-\mathrm{C}_{\alpha}-\mathrm{O}_{\alpha}$ and $\mathrm{C}_{\beta}-\mathrm{C}_{\alpha}-\mathrm{O}_{\alpha}-\mathrm{P}$ dihedrals of the PS and PC headgroups. The trajectory used for CHARMM36 POPC is available at Ref. 117. 
Interestingly, in the three models that best fit the experimental data, the $\mathrm{C}_{\alpha}-\mathrm{C}_{\beta}-\mathrm{C}_{\gamma}-\mathrm{O}_{\gamma}$ dihedral angle distribution has a single peak around $120^{\circ}$, while the other models yield binodal distributions (Fig. S7). The restricted motion is also visible in the sampled conformations (Figs. 5 (A) and S9), suggesting that the rotation of the carboxyl group is limited in the serine headgroup. On the other hand, the $\mathrm{C}_{\beta}-\mathrm{C}_{\alpha^{-}} \mathrm{O}_{\alpha}-\mathrm{P}$ dihedral angle rotates relatively freely between approximately $100^{\circ}$ and $300^{\circ}$ in the best three models (as seen also in Fig. 5 (B)), while other models yield more restricted conformations (Fig. S7).

In simulations that have the best agreement with experiments (CHARMM36 and Slipids), the $\mathrm{N}-\mathrm{C}_{\beta}-\mathrm{C}_{\alpha}-\mathrm{O}_{\alpha}$ dihedral exhibits a more asymmetric angle distribution for PS than for PC headgroup (Figs. 5 (B) and S10), which might reflect the increased rigidity proposed in the early experimental studies. ${ }^{7,8}$ Indeed, the characteristic conformations of the PS headgroup suggested here can be useful when interpreting experiments - however, as none of the tested models fully reproduces the experimental order parameters, more accurate MD force fields are required to reveal the true conformational ensemble.

\section{Counterion binding and interactions between PC and PS head- groups}

Membranes containing PS lipids are always accompanied with counterions that modulate electrostatic interactions between the lipids and other biomolecules. MD simulations have suggested that counterions reduce the area per lipid of PS bilayers compared to PC bilayers ${ }^{23-25}$ by screening the repulsion between charged lipid headgroups. We explored this by quantifying the counterion density profiles along the membrane normal, accompanied by the areas per lipid, $\left\langle A_{\text {pl }}\right\rangle$, see Fig. 6. The force fields studied showed significant differences in both the binding affinity and the distribution of ions at the interface. The experimental area per lipid $\left(62.7 \pm 1.3 \AA^{2}\right)^{30}$ was reproduced only in GROMOS-CKP and GROMOS-CKPM, as well as in MacRog with potassium counterions, while all other models gave considerably smaller values (Fig. 6). However, the counterion binding and the concomitant electrostatic screening 
of the headgroup repulsion does not fully explain the low area per lipid values since MacRog, which has the strongest sodium binding (the lowest concentrations in bulk water), gave the same $\left\langle A_{\mathrm{pl}}\right\rangle$ as CHARMM36-UA, which has significantly weaker counterion binding affinity. On the other hand, MacRog simulation with potassium produced a larger area per lipid $\left(63 \AA^{2}\right)$ than with sodium $\left(53 \AA^{2}\right)$, in line with the weaker potassium binding affinity (Fig. 6). The results are consistent with a previous study that suggested the low $\left\langle A_{\mathrm{pl}}\right\rangle$ in PS lipid bilayers to originate from the combination of both the counterion binding and intermolecular interactions between lipid headgroups. ${ }^{118}$

The experimentally observed modulation of headgroup order parameters by increasing salt concentration (the molecular electrometer concept) has been previously used to evaluate cation binding to zwitterionic PC bilayers in simulations. ${ }^{39}$ Studying the binding of cations to negatively charged lipid bilayers is less straightforward, because an ion-free reference state does not exist due to the ever-present cationic counterions. Our analysis was further complicated by the artificial aggregation of counterions observed in some simulations (section S7 in the Supporting Information). Therefore, we evaluate here the amount of bound charge not by adding salt (although this is discussed in section S7), but by studying the changes of the headgroup $S_{\mathrm{CH}}$ with an increasing amount of negatively charged lipids (and thus an increasing amount of cationic counterions) in the bilayer.

Experimentally, the $S_{\mathrm{CH}}$ values of the $\alpha$ and $\beta$ headgroup carbons of POPC increase when negatively charged POPS lipids are incorporated in the bilayer (section S2). ${ }^{43,107}$ This was reproduced in the MacRog simulations with potassium counterions (Fig. 7), which had the weakest binding affinity to POPS lipid bilayers (Fig. 6). The CHARMM36, Berger, and GROMOS-CKP simulations either exhibited no change, or showed a decrease in one or even both of the POPC headgroup order parameters, as the amount of POPS increased (Fig. 7). Therein, the stronger counterion binding canceled the effect of negatively charged headgroups and prevented the experimentally observed increase of headgroup order parameters with growing amount of PS lipids. Therefore, we suggest that the relatively weak binding of 


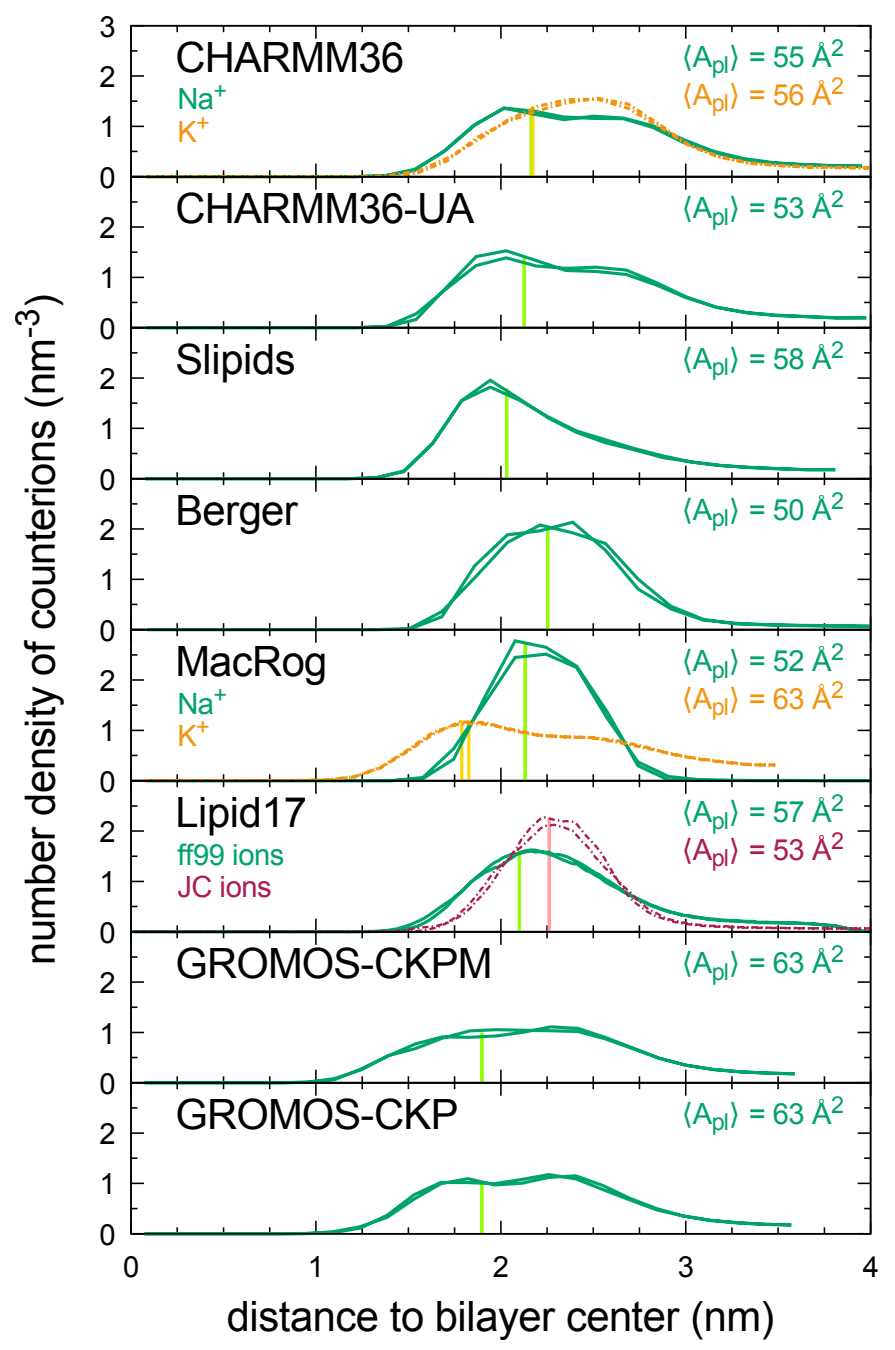

Figure 6: Counterion density profiles along the membrane normal and areas per lipid, $\left\langle A_{\mathrm{pl}}\right\rangle$, of POPS lipid bilayer from simulations with different force fields. The vertical bars indicate the locations of the phosphate density peaks. The experimental area per lipid is $62.7 \pm 1.3 \AA^{2}{ }^{30}$

potassium in the MacRog simulations (Fig. 6) produces a more realistic surface charge density in membranes containing PS lipids than the other tested models that overestimate the counterion binding affinity. The results are consistent with the behavior of headgroup order parameters as a function of added counterions, see section S7 in the Supporting Information.

The reduced forking of the POPS $\alpha$-carbon (Fig. 8) together with other experimental results suggest that the PS headgroup structure becomes less rigid when diluted with 

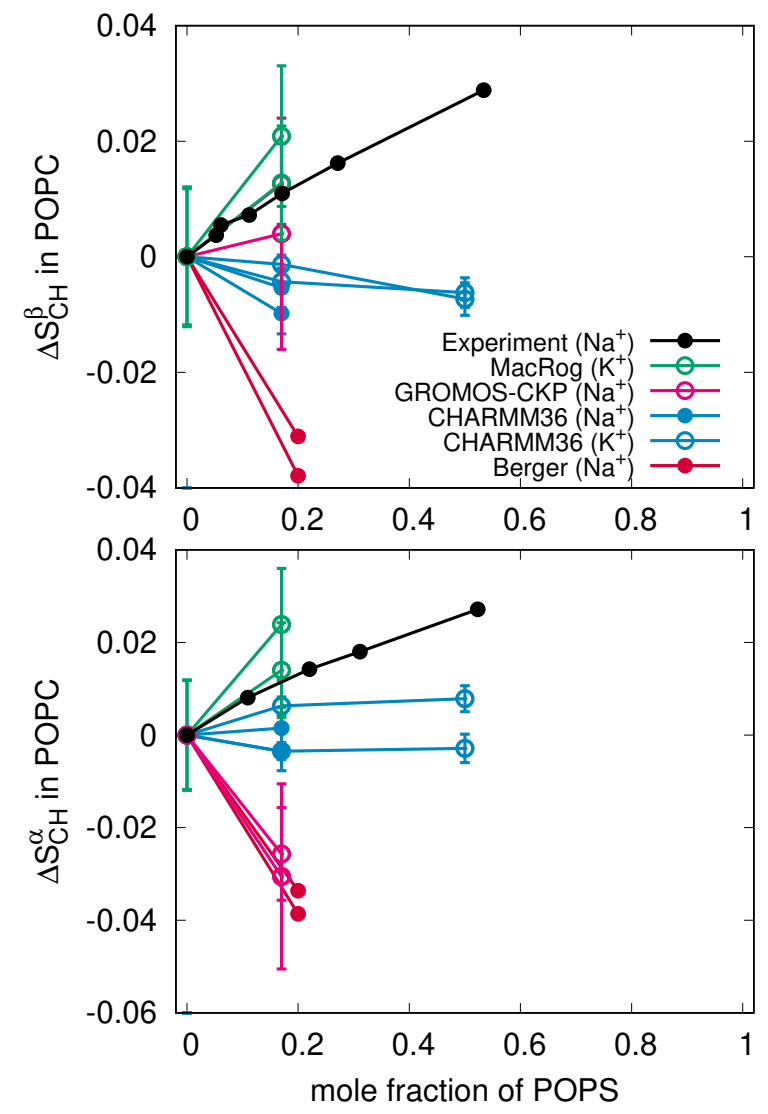

Figure 7: Changes of POPC headgroup order parameters with increasing amount of POPS in POPC:POPS mixtures at $298 \mathrm{~K}$. Experimental values are from Ref. 43, with the signs measured in Ref. 47. The values from the CHARMM36 and GROMOS-CKP simulations with sodium are averages of two independent simulations, the error bars show the difference between the results divided by two.

POPC. ${ }^{7,8,17,18,43}$ Unfortunately, none of the tested models correctly reproduced the modulation of POPS headgroup order parameters with increasing amount of POPC (Fig. 8). More accurate force fields are needed to correctly describe the PC-PS headgroup interactions in MD simulations.

\section{$\mathrm{Ca}^{2+}$ binding affinity to bilayers with negatively charged PS lipids}

Calcium binding affinity to membranes containing negatively charged PS lipids can be experimentally quantified by measuring the PC lipid headgroup order parameters from POPC:POPS (5:1) mixtures (see section S2), where the measurement is not compromised by the dehydrated 

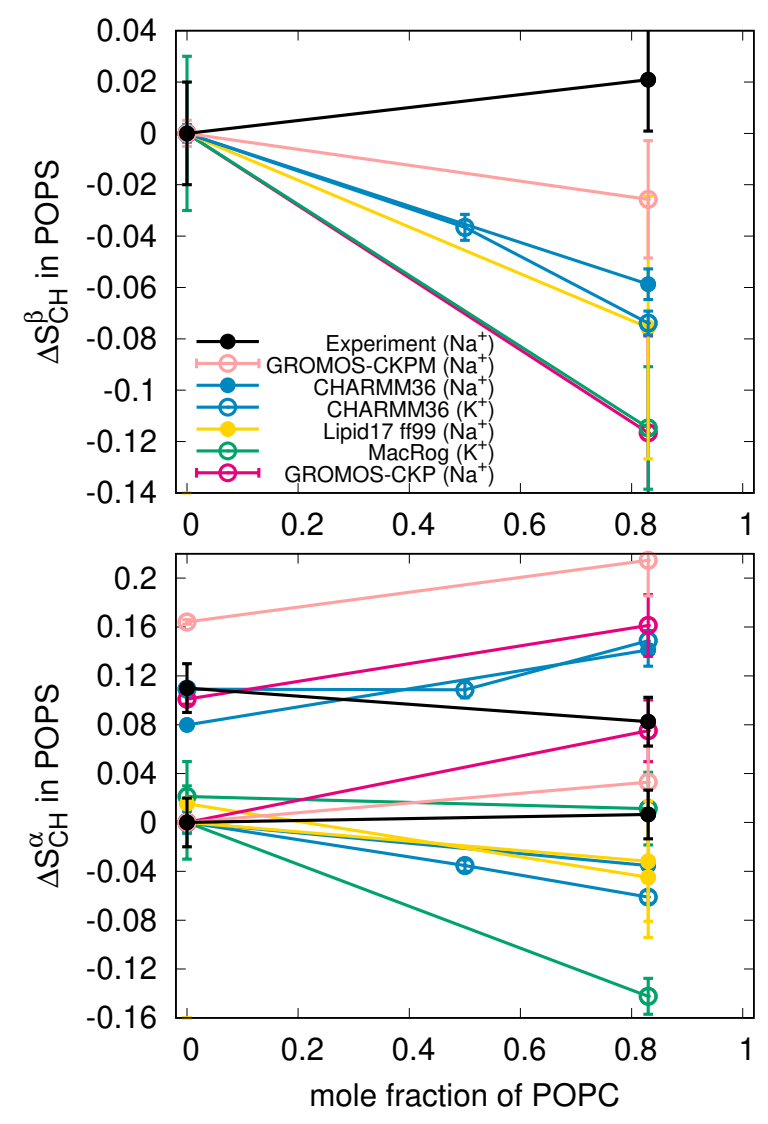

Figure 8: Modulation of the POPS headgroup order parameters with increasing amount of POPC in POPC:POPS mixtures at $298 \mathrm{~K}$. Experimental values with the signs were measured for a pure POPS system in this work. The signs are assumed to remain unchanged for the mixture, and the magnitudes are taken from Ref. 17. Because the experimental values of POPS in pure and mixed bilayers come from ${ }^{13} \mathrm{C}$ NMR (this work) and ${ }^{2} \mathrm{H}$ NMR (Ref. 17), respectively, the error bars of 0.02 are used here. ${ }^{38,40}$ The $y$-axis for the $\alpha$-carbon results (bottom) is shifted with the same value for both order parameters such that the lower order parameter value from pure POPS is at zero to correctly illustrate the significant forking. The values from CHARMM36 and GROMOS simulations are averages of two independent simulations, the error bars show the difference between the results divided by two.

lipid-ion complexes and phase separation, and the bilayer remains uniform. ${ }^{15-18}$ Despite the lack of an ion-free reference state in the presence of negatively charged lipids, our simulations gave coherent results for the POPC headgroup order parameters as a function of $\mathrm{CaCl}_{2}$ concentration in POPC:POPS (5:1) mixtures (Fig. 9). As expected from the previous study of pure PC lipid bilayers, ${ }^{39}$ almost all the tested simulation models overestimated the experimentally observed ${ }^{17}$ decrease of the POPC headgroup $S_{\mathrm{CH}}$ upon increasing $\mathrm{Ca}^{2+}$ 
POPC
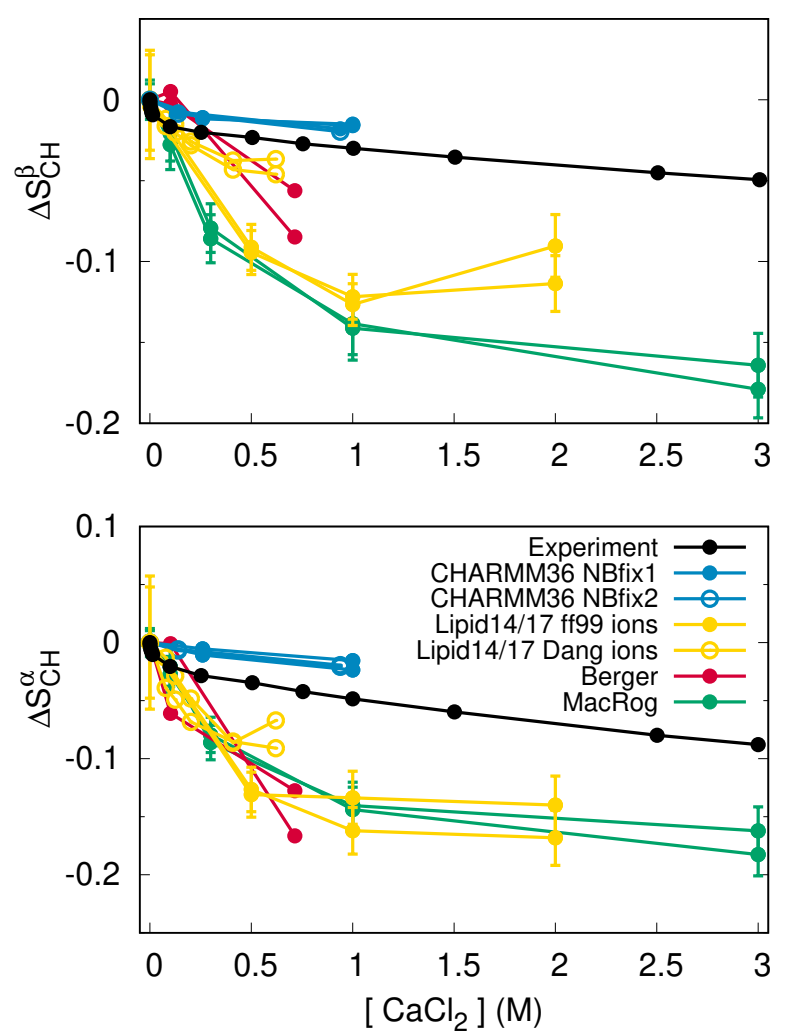

POPS
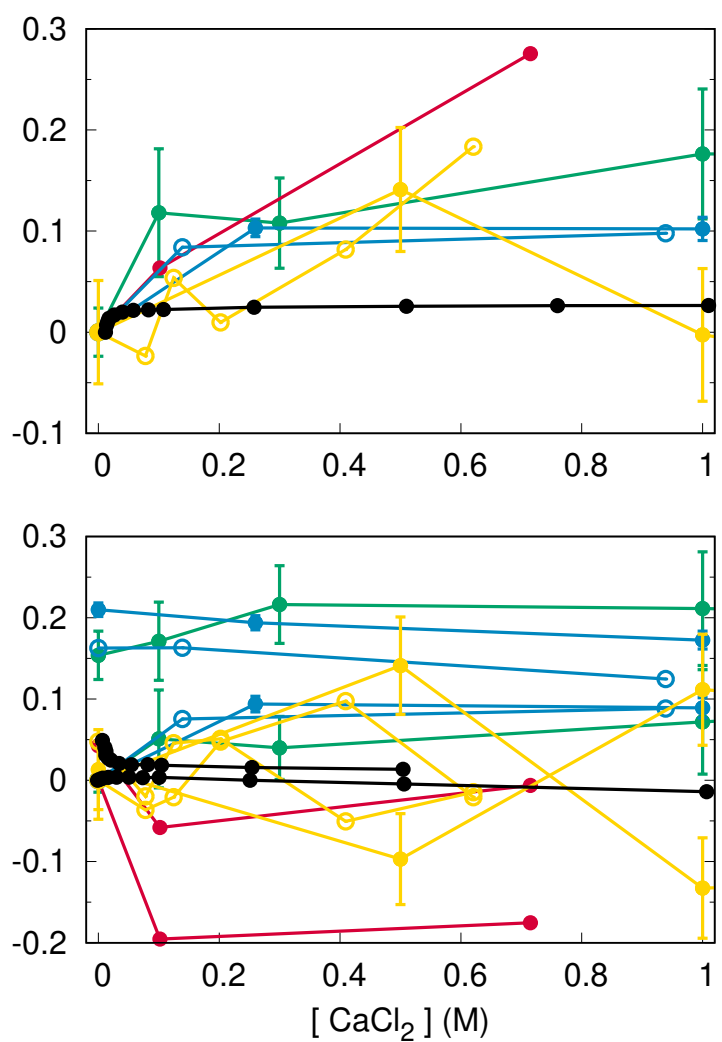

Figure 9: Variation of the POPC (left) and POPS (right) headgroup order parameters in a POPC:POPS (5:1) mixture as a function $\mathrm{CaCl}_{2}$ concentration from experiments ${ }^{17}$ and from different force fields at $298 \mathrm{~K}$ (except the data for Berger are from a simulation of a POPC:POPS (4:1) mixture at $\left.310 \mathrm{~K}^{32,119}\right)$. The $S_{\mathrm{CH}}$ values from systems without calcium are set at the zero point of y-axes, except for the $\alpha$-carbon order parameters of POPS (bottom right), for which both $S_{\mathrm{CH}}^{\alpha}$ are shifted such that the lower $S_{\mathrm{CH}}^{\alpha}$ is zero without additional ions. This is to correctly illustrate the forking with different concentrations of calcium. Counterions were potassium in MacRog simulations, and sodium in Lipid14/17 Dang and CHARMM36 NBfix2 simulations. In CHARMM36 NBfix1, Lipid14/17 ff99, and Berger simulations with added calcium the lipid charge was neutralized with calcium, and monovalent counterions were not present; for these systems the $x$-axis shows $\left[\mathrm{Ca}^{2+}\right]$. 


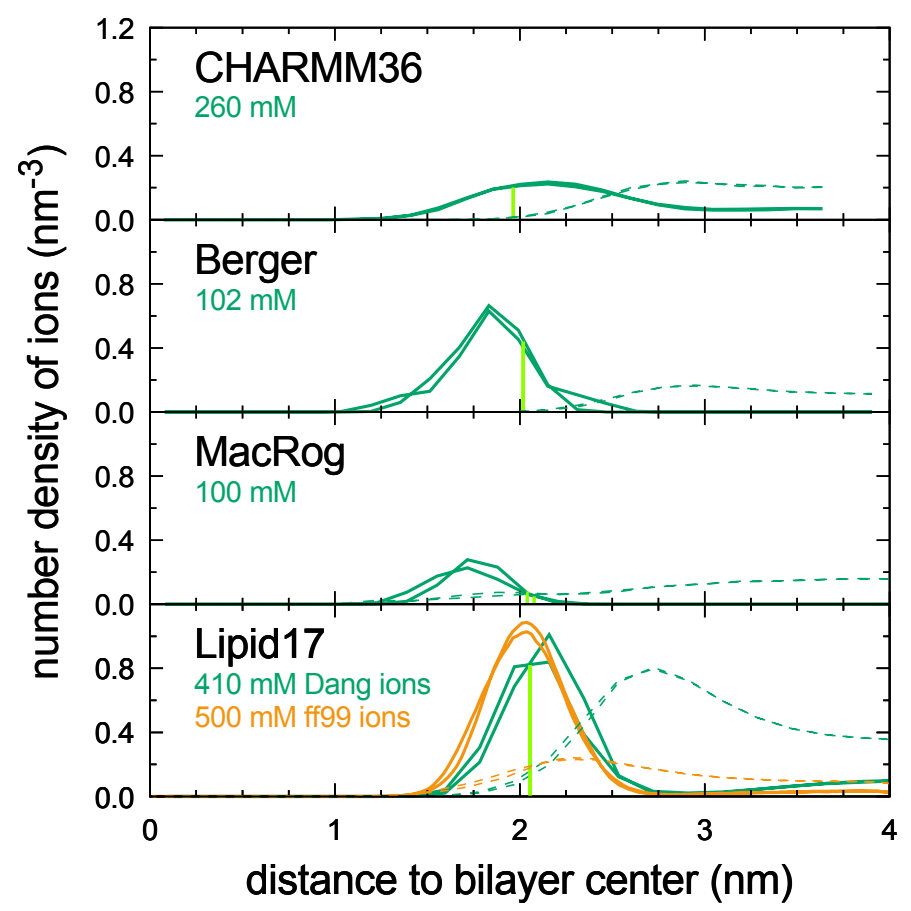

Figure 10: Number density profiles of $\mathrm{Ca}^{2+}$ (solid line) and $\mathrm{Cl}^{-}$(dashed line) from POPC:POPS (5:1) mixtures simulated with different force fields. The vertical green bars indicate the location of the phosphate density peak. The smallest simulated $\mathrm{CaCl}_{2}$ concentrations are shown. The density profiles for all the simulated concentrations are given in SI figure S18.

concentration (Fig. 9), indicating too strong calcium binding affinity. The sole exception was CHARMM36 when paired with the NBfix corrections for calcium; ${ }^{35,87}$ for these combinations, the modulation of order parameters was underestimated, indicating a weaker binding affinity than in experiments. Notably, CHARMM36 with the NBfix corrections ${ }^{29,35}$ suggested similar binding affinities for calcium and sodium to a POPC bilayer (see section S8), in contrast to experiments. ${ }^{105,106,120}$ This suggests that the calcium binding affinity is underestimated in CHARMM36 when using the NBfix for calcium, ${ }^{35,87}$ but overestimated in all the other tested force fields. This is evident in the calcium density distributions, where almost all the $\mathrm{Ca}^{2+}$ ions bind to the membrane interface in all models except CHARMM36 (Fig. 10).

Experimentally, the POPS headgroup order parameters in POPC:POPS (5:1) mixtures exhibit a strong response to small concentrations of $\mathrm{CaCl}_{2}$, which saturates below $100 \mathrm{mM}$ (Fig. 9). The $\beta$-carbon $S_{\mathrm{CH}}$ increases with added $\mathrm{CaCl}_{2}$, whereas the larger $\alpha$-carbon 
$S_{\mathrm{CH}}$ decreases. Moreover, a slight increase is observed in the smaller $\alpha$-carbon $S_{\mathrm{CH}}$. In simulations, all these responses were qualitatively correct only in CHARMM36. In all force fields, even the qualitatively correct responses were much exaggerated - also in CHARMM36 that underestimated the $\mathrm{Ca}^{2+}$ binding affinity - with the sole exception of the larger $S_{\mathrm{CH}}^{\alpha}$, whose change CHARMM36 underestimated. Importantly, different force fields predicted qualitatively different behavior for the two POPS $\alpha$-carbon order parameters as a function of added calcium: Both order parameters decreased in Berger, but increased in MacRog, whereas Lipid14/17 and CHARMM36 models exhibited more complex behaviors. This is in contrast to the $\mathrm{PC}$ headgroup, where a qualitatively correct response to bound ions is reproduced by all simulation models, despite the significant discrepancies in the headgroup structures observed in salt-free simulations. ${ }^{39}$ The divergent response of Berger may arise from the ring-like structures observed in the headgroup region in this model (Fig. 6 in Ref. 24). Therefore, we conclude that improvement of force fields is necessary to correctly capture the interactions between the PS headgroup and calcium ions in MD simulations.

\section{Conclusions}

We used the headgroup C-H bond order parameters, $S_{\mathrm{CH}}$, and the open collaboration approach to evaluate the quality of the headgroup structure and the ion binding affinity in available MD models of PS lipids. The main advantage of using the $S_{\mathrm{CH}}$ is the direct connection they provide between experiments and simulations: They can be measured accurately in experiments and calculated readily from simulations. This reduces the ambiguity in the interpretation of experiments.

First, we complemented the available experimental information ${ }^{7,17}$ by measuring the signs of the PS headgroup order parameters, and then proceeded to compare MD simulation results from several force fields with the experimental data. This revealed that none of the force fields reproduce the PS headgroup order parameters within the experimental accuracy. 
However, the best models captured essential differences between PS and PC, and suggested characteristic conformations of PS headgroups. Comparison to the experimentally observed order parameters in POPC:POPS (5:1) bilayers at varying ion concentrations ${ }^{17}$ then showed that the tested MD force fields overestimate the cation binding affinity to these bilayers. There were two exceptions: 1) the MacRog force field with potassium counterions, which appeared to display a more realistic monovalent ion binding affinity to PS-containing lipid bilayers than the other models; and 2) the CHARMM36 force field with the recently introduced NBfix corrections for calcium, ${ }^{35,87}$ which underestimated the calcium binding affinity. Importantly, the experimentally measured responses of the PS headgroup $S_{\mathrm{CH}}$ to bound calcium, as well as to dilution of the bilayer with zwitterionic PC lipids, were not qualitatively reproduced in any of the tested force fields. This is in contrast to previous results with PC lipids, where MD force fields were seen to be at least in qualitative agreement with the experimentally measured headgroup $S_{\mathrm{CH}}$ responses to bound charge - even though the headgroup structures themselves were incorrect and the cation binding affinities were overestimated. ${ }^{39}$ This underlines the dire need for more realistic MD force fields to study the biological function of PS lipids.

We expect our results to pave the way for the development of better PS force fields. As the quality of the conformational ensembles can be evaluated against the headgroup $\mathrm{C}-\mathrm{H}$ order parameters, we hope that the $S_{\mathrm{CH}}$ will guide the development of models that correctly reproduce the PS headgroup structures. For example, the ensembles we observed in the simulations with the most realistic headgroup conformations (CHARMM36 and Slipids) do hint a direction for force field improvement. The cation binding could be improved based on the experimental headgroup $S_{\mathrm{CH}}$ data from POPC:POPS (5:1) mixtures under different cation concentrations. Recent studies have shown that cation binding to bilayers with POPC and POPS lipids can be improved by implicit inclusion of electronic polarizability using the electronic continuum correction (ECC) ${ }^{112,121}$ suggesting that the electronic polarizability effects are important for lipid-ion interactions but polarizable force fields may not be necessary to correctly capture ion binding to lipid bilayers. 


\section{Supporting Information Available}

The following files are available free of charge.

- SI.pdf: Additional simulation, experimental and methodological details, and further analysis.

\section{Acknowledgement}

HA acknowledges the Osk. Huttunen Foundation and the Finnish Academy of Science and Letters (Foundations' Post Doc Pool) for financial support. FFR acknowledges Tecnológico Nacional de México Campus Zacatecas Occidente and Dirección General de Asuntos del Personal Académico (DGAPA) Programa de Apoyo a Proyectos de Investigación e Innovación Tecnológica (PAPIIT) IG100416 for financial support and Clúster Híbrido de Supercómputo Xiuhcoatl-CINVESTAV and Miztli-Dirección de Cómputo y de Tecnologías de Información

y Comunicación (DGTIC)-Universidad Nacional Autónoma de México (UNAM) (Project LANCAD-UNAM-DGTIC-028) facilities for computing-time allocation. MJ acknowledges financial support from the Emil Aaltonen Foundation and CSC - IT Center for Science for computational resources. BK gratefully acknowledges financial support from the International Max Planck Research School (IMPRS) on Multiscale Bio-Systems. JJM gratefully acknowledges financial support from the Carlsberg Foundation in the form of a postdoctoral fellowship while at the University of Chicago (grants CF15-0552, CF16-0639, and CF17-0783) and the research framework provided by the Research Computing Center at the University of Chicago. OHSO acknowledges financial support from Academy of Finland (315596), Integrated Structural Biology Research Infrastructure of Helsinki Institute of Life Science (Instruct-HiLIFE), and CSC - IT Center for Science for computational resources. TJP wishes to acknowledge the use of the Iridis computing resources at the University of Southampton. 


\section{References}

(1) Lemmon, M. A. Membrane Recognition by Phospholipid-Binding Domains. Nat. Rev. Mol. Cell Biol. 2008, 9, 99-111.

(2) Leventis, P. A.; Grinstein, S. The Distribution and Function of Phosphatidylserine in Cellular Membranes. Annu. Rev. Biophys. 2010, 39, 407-427.

(3) Li, L.; Shi, X.; Guo, X.; Li, H.; Xu, C. Ionic Protein-Lipid Interaction at the Plasma Membrane: What Can the Charge Do? Trends Biochem. Sci 2014, 39, 130 - 140.

(4) Yeung, T.; Gilbert, G. E.; Shi, J.; Silvius, J.; Kapus, A.; Grinstein, S. Membrane Phosphatidylserine Regulates Surface Charge and Protein Localization. Science 2008, 319, 210-213.

(5) Zhao, H.; Tuominen, E. K. J.; Kinnunen, P. K. J. Formation of Amyloid Fibers Triggered by Phosphatidylserine-Containing Membranes. Biochemistry 2004, 43, 10302-10307.

(6) Gorbenko, G. P.; Kinnunen, P. K. the Role of Lipid-Protein Interactions in AmyloidType Protein Fibril Formation. Chem. Phys. Lipids 2006, 141, $72-82$.

(7) Browning, J. L.; Seelig, J. Bilayers of Phosphatidylserine: A Deuterium and Phosphorus Nuclear Magnetic Resonance Study. Biochemistry 1980, 19, 1262-1270.

(8) Büldt, G.; Wohlgemuth, R. the Headgroup Conformation of Phospholipids in Membranes. J. Membr. Biol. 1981, 58, 81-100.

(9) Hauser, H.; Finer, E.; Darke, A. Crystalline Anhydrous Ca-Phosphatidylserine Bilayers. Biochem. Biophys. Res. Commun. 1977, 76, $267-274$.

(10) Kurland, R. J. Binding of Ca2+ and Mg2+ to Phosphatidylserine Vesicles: Different Effects on P-31 NMR Shifts and Relaxation Times. Biochem. Biophys. Res. Commun. $\mathbf{1 9 7 9}, 88,927-932$. 
(11) Eisenberg, M.; Gresalfi, T.; Riccio, T.; McLaughlin, S. Adsorption of Monovalent Cations to Bilayer Membranes Containing Negative Phospholipids. Biochemistry 1979, $18,5213-5223$.

(12) Hauser, H.; Shipley, G. G. Interactions of Monovalent Cations with Phosphatidylserine Bilayer Membranes. Biochemistry 1983, 22, 2171-2178.

(13) Dluhy, R.; Cameron, D. G.; Mantsch, H. H.; Mendelsohn, R. Fourier Transform Infrared Spectroscopic Studies of the Effect of Calcium Ions on Phosphatidylserine. Biochemistry 1983, 22, 6318-6325.

(14) Hauser, H.; Shipley, G. Comparative Structural Aspects of Cation Binding to Phosphatidylserine Bilayers. Biochim. Biophys. Acta 1985, 813, $343-346$.

(15) Feigenson, G. W. On the Nature of Calcium Ion Binding Between Phosphatidylserine Lamellae. Biochemistry 1986, 25, 5819-5825.

(16) Mattai, J.; Hauser, H.; Demel, R. A.; Shipley, G. G. Interactions of Metal Ions with Phosphatidylserine Bilayer Membranes: Effect of Hydrocarbon Chain Unsaturation. Biochemistry 1989, 28, 2322-2330.

(17) Roux, M.; Bloom, M. Calcium, Magnesium, Lithium, Sodium, and Potassium Distributions in the Headgroup Region of Binary Membranes of Phosphatidylcholine and Phosphatidylserine As Seen by Deuterium NMR. Biochemistry 1990, 29, 7077-7089.

(18) Roux, M.; Bloom, M. Calcium Binding by Phosphatidylserine Headgroups. Deuterium NMR Study. Biophys. J. 1991, 60, $38-44$.

(19) Boettcher, J. M.; Davis-Harrison, R. L.; Clay, M. C.; Nieuwkoop, A. J.; Ohkubo, Y. Z.; Tajkhorshid, E.; Morrissey, J. H.; Rienstra, C. M. Atomic View of Calcium-Induced Clustering of Phosphatidylserine in Mixed Lipid Bilayers. Biochemistry 2011, 50, $2264-2273$. 
(20) Seelig, J. Interaction of Phospholipids with Ca2+ Ions. on the Role of the Phospholipid Head Groups. Cell Biol. Int. Rep. 1990, 14, 353 - 360.

(21) Sinn, C. G.; Antonietti, M.; Dimova, R. Binding of Calcium to PhosphatidylcholinePhosphatidylserine Membranes. Colloids Surf., A 2006, 282-283, 410 - 419.

(22) López Cascales, J. J.; García de la Torre, J.; Marrink, S. J.; Berendsen, H. J. C. Molecular Dynamics Simulation of a Charged Biological Membrane. J. Chem. Phys. 1996, 104, 2713-2720.

(23) Pandit, S. A.; Berkowitz, M. L. Molecular Dynamics Simulation of Dipalmitoylphosphatidylserine Bilayer with Na+ Counterions. Biophys. J. 2002, 82, 1818 - 1827.

(24) Mukhopadhyay, P.; Monticelli, L.; Tieleman, D. P. Molecular Dynamics Simulation of a Palmitoyl-Oleoyl Phosphatidylserine Bilayer with $\mathrm{Na}+$ Counterions and $\mathrm{NaCl}$. Biophys. J. 2004, 86, $1601-1609$.

(25) Pedersen, U. R.; Leidy, C.; Westh, P.; Peters, G. H. the Effect of Calcium on the Properties of Charged Phospholipid Bilayers. Biochim. Biophys. Acta 2006, 1758, 573 $-582$.

(26) Vernier, P. T.; Ziegler, M. J.; Dimova, R. Calcium Binding and Head Group Dipole Angle in Phosphatidylserine-Phosphatidylcholine Bilayers. Langmuir 2009, 25, 10201027.

(27) Martín-Molina, A.; Rodríguez-Beas, C.; Faraudo, J. Effect of Calcium and Magnesium on Phosphatidylserine Membranes: Experiments and All-Atomic Simulations. Biophys. J. 2012, 102, $2095-2103$.

(28) Jurkiewicz, P.; Cwiklik, L.; Vojtíšková, A.; Jungwirth, P.; Hof, M. Structure, Dynamics, and Hydration of POPC/POPS Bilayers Suspended in $\mathrm{NaCl}, \mathrm{KCl}$, and $\mathrm{CsCl}$ Solutions. Biochim. Biophys. Acta 2012, 1818, $609-616$. 
(29) Venable, R. M.; Luo, Y.; Gawrisch, K.; Roux, B.; Pastor, R. W. Simulations of Anionic Lipid Membranes: Development of Interaction-Specific Ion Parameters and Validation Using NMR Data. J. Phys. Chem. B 2013, 117, 10183-10192.

(30) Pan, J.; Cheng, X.; Monticelli, L.; Heberle, F. A.; Kucerka, N.; Tieleman, D. P.; Katsaras, J. the Molecular Structure of a Phosphatidylserine Bilayer Determined by Scattering and Molecular Dynamics Simulations. Soft Matter 2014, 10, 3716-3725.

(31) Vangaveti, S.; Travesset, A. Separation of the Stern and Diffuse Layer in CoarseGrained Models: The Cases of Phosphatidyl Serine, Phosphatidic Acid, and PIP2 Monolayers. J. Chem. Phys. 2014, 141, 245102.

(32) Melcrová, A.; Pokorna, S.; Pullanchery, S.; Kohagen, M.; Jurkiewicz, P.; Hof, M.; Jungwirth, P.; Cremer, P. S.; Cwiklik, L. the Complex Nature of Calcium Cation Interactions with Phospholipid Bilayers. Sci. Reports 2016, 6, 38035.

(33) Valentine, M. L.; Cardenas, A. E.; Elber, R.; Baiz, C. R. Physiological Calcium Concentrations Slow Dynamics at the Lipid-Water Interface. Biophys. J. 2018, 115, $1541-1551$.

(34) Hallock, M. J.; Greenwood, A. I.; Wang, Y.; Morrissey, J. H.; Tajkhorshid, E.; Rienstra, C. M.; Pogorelov, T. V. Calcium-Induced Lipid Nanocluster Structures: Sculpturing of the Plasma Membrane. Biochemistry 2018, 57, 6897-6905.

(35) Kim, S.; Patel, D. S.; Park, S.; Slusky, J.; Klauda, J. B.; Widmalm, G.; Im, W. Bilayer Properties of Lipid a from Various Gram-Negative Bacteria. Biophys. J. 2016, 111, $1750-1760$.

(36) Klauda, J. B.; Venable, R. M.; Freites, J. A.; O'Connor, J. W.; Tobias, D. J.; MondragonRamirez, C.; Vorobyov, I.; MacKerell Jr, A. D.; Pastor, R. W. Update of the CHARMM All-Atom Additive Force Field for Lipids: Validation on Six Lipid Types. J. Phys. Chem. B 2010, 114, 7830-7843. 
(37) Berger, O.; Edholm, O.; Jähnig, F. Molecular Dynamics Simulations of a Fluid Bilayer of Dipalmitoylphosphatidylcholine at Full Hydration, Constant Pressure, and Constant Temperature. Biophys. J. 1997, 72, $2002-2013$.

(38) Botan, A.; Favela-Rosales, F.; Fuchs, P. F. J.; Javanainen, M.; Kanduč, M.; Kulig, W.; Lamberg, A.; Loison, C.; Lyubartsev, A.; Miettinen, M. S. et al. Toward Atomistic Resolution Structure of Phosphatidylcholine Headgroup and Glycerol Backbone at Different Ambient Conditions. J. Phys. Chem. B 2015, 119, 15075-15088.

(39) Catte, A.; Girych, M.; Javanainen, M.; Loison, C.; Melcr, J.; Miettinen, M. S.; Monticelli, L.; Maatta, J.; Oganesyan, V. S.; Ollila, O. H. S. et al. Molecular Electrometer and Binding of Cations to Phospholipid Bilayers. Phys. Chem. Chem. Phys. 2016, 18, $32560-32569$.

(40) Ollila, O. S.; Pabst, G. Atomistic Resolution Structure and Dynamics of Lipid Bilayers in Simulations and Experiments. Biochim. Biophys. Acta 2016, 1858, $2512-2528$.

(41) Piggot, T. J.; Allison, J. R.; Sessions, R. B.; Essex, J. W. On the Calculation of Acyl Chain Order Parameters from Lipid Simulations. J. Chem. Theory Comput. 2017, 13, $5683-5696$.

(42) Gally, H. U.; Pluschke, G.; Overath, P.; Seelig, J. Structure of Escherichia Coli Membranes. Glycerol Auxotrophs As a Tool for the Analysis of the Phospholipid HeadGroup Region by Deuterium Magnetic Resonance. Biochemistry 1981, 20, 1826-1831.

(43) Scherer, P.; Seelig, J. Structure and Dynamics of the Phosphatidylcholine and the Phosphatidylethanolamine Head Group in L-M Fibroblasts As Studied by Deuterium Nuclear Magnetic Resonance. EMBO J. 1987, 6, 2915-2922.

(44) Dvinskikh, S. V.; Zimmermann, H.; Maliniak, A.; Sandstrom, D. Measurements of Motionally Averaged Heteronuclear Dipolar Couplings in MAS NMR Using R-Type Recoupling. J. Magn. Reson. 2004, 168, 194-201. 
(45) Gross, J. D.; Warschawski, D. E.; Griffin, R. G. Dipolar Recoupling in MAS NMR: A Probe for Segmental Order in Lipid Bilayers. J. Am. Chem. Soc. 1997, 119, 796-802.

(46) Ferreira, T. M.; Coreta-Gomes, F.; Ollila, O. H. S.; Moreno, M. J.; Vaz, W. L. C.; Topgaard, D. Cholesterol and POPC Segmental Order Parameters in Lipid Membranes: Solid State ${ }^{1} \mathrm{H}-{ }^{13} \mathrm{C}$ NMR and MD Simulation Studies. Phys. Chem. Chem. Phys. 2013, $15,1976-1989$.

(47) Ferreira, T. M.; Sood, R.; Bärenwald, R.; Carlström, G.; Topgaard, D.; Saalwächter, K.; Kinnunen, P. K. J.; Ollila, O. H. S. Acyl Chain Disorder and Azelaoyl Orientation in Lipid Membranes Containing Oxidized Lipids. Langmuir 2016, 32, 6524-6533.

(48) Bak, M.; Rasmussen, J. T.; Nielsen, N. C. SIMPSON: A General Simulation Program for Solid-State NMR Spectroscopy. J. Magn. Res. 2000, 147, 296 - 330.

(49) Ollila, O. H. S.; Kav, B.; Antila, H.; Peon, A.; Miettinen, M.; Melcr, J.; Määttä, J.; Buslaev, P. NMRLipids MATCH Repository Databank. 2019; https://doi.org/10. 5281 /zenodo. 3372345 .

(50) Joung, I. S.; Cheatham, T. E. Determination of Alkali and Halide Monovalent Ion Parameters for Use in Explicitly Solvated Biomolecular Simulations. J. Phys. Chem. B 2008, 112, 9020-9041.

(51) Åqvist, J. Ion-Water Interaction Potentials Derived from Free Energy Perturbation Simulations. J. Phys. Chem. 1990, 94, 8021-8024.

(52) Piggot, T. CHARMM36 POPS Simulations (versions 1 and 2) 298 K 1.0nmLJ Switching. 2017; https://doi.org/10.5281/zenodo. 1129415.

(53) Piggot, T. CHARMM36 POPS Simulations (versions 1 and 2) 298 K 1.0nmLJ Switching with K Ions. 2018; https://doi.org/10.5281/zenodo.1182654. 
(54) Lee, S.; Tran, A.; Allsopp, M.; Lim, J. B.; Henin, J.; Klauda, J. B. CHARMM36 United Atom Chain Model for Lipids and Surfactants. J. Phys. Chem. B 2014, 118, 547-556.

(55) Piggot, T. CHARMM36-Ua POPS Simulations (versions 1 and 2) 298 K 1.0nmLJ Switching. 2017; https://doi.org/10.5281/zenodo.1129458.

(56) Maciejewski, A.; Pasenkiewicz-Gierula, M.; Cramariuc, O.; Vattulainen, I.; Róg, T. Refined OPLS All-Atom Force Field for Saturated Phosphatidylcholine Bilayers at Full Hydration. J. Phys. Chem. B 2014, 118, 4571-4581.

(57) Piggot, T. MacRog POPS Simulations (versions 1 and 2) $298 \mathrm{~K}$ with Corrected PO Not OP Tails. 2018; https://doi.org/10.5281/zenodo.1283335.

(58) Javanainen, M. Simulation of a POPS Membrane with Potassium Counterions. 2018; https://doi.org/10.5281/zenodo. 1434990.

(59) Gould, I.; Skjevik, A.; Dickson, C.; Madej, B.; Walker, R. Lipid17: A Comprehensive AMBER Force Field for the Simulation of Zwitterionic and Anionic Lipids. 2018; In preparation.

(60) Miettinen, M. S.; Kav, B. Molecular Dynamics Simulation Trajectory of an Anionic Lipid Bilayer: 100 Mol\% POPS with Na+ Counterions Using Joung-Cheatham Ions. 2018; https://doi.org/10.5281/zenodo.1148495.

(61) Miettinen, M. S.; Kav, B. Molecular Dynamics Simulation Trajectory of an Anionic Lipid Bilayer: 100 Mol\% POPS with Na+ Counterions Using Ff99 Ions. 2018; https: //doi.org/10.5281/zenodo.1134869.

(62) Piggot, T. Berger POPS Simulations (versions 1 and 2) 298 K 1.0nmCut-Off. 2017; https://doi.org/10.5281/zenodo. 1129425.

(63) Chandrasekhar, I.; Kastenholz, M.; Lins, R.; Oostenbrink, C.; Schuler, L.; Tieleman, D.; Gunsteren, W. A Consistent Potential Energy Parameter Set for Lipids: Dipalmi- 
toylphosphatidylcholine As a Benchmark of the GROMOS96 45A3 Force Field. Eur. Biophys. J. 2003, 32, 67-77.

(64) Kukol, A. Lipid Models for United-Atom Molecular Dynamics Simulations of Proteins. J. Chem. Theory Comput. 2009, 5, 615-626.

(65) Piggot, T. J.; Piñeiro, Á.; Khalid, S. Molecular Dynamics Simulations of Phosphatidylcholine Membranes: A Comparative Force Field Study. J. Chem. Theory Comput. 2012, 8, 4593-4609.

(66) Piggot, T. GROMOS-CKP POPS Simulations (versions 1 and 2) $298 \mathrm{~K}$ with Berger/Chiu NH3 Charges and PME. 2017; https://doi.org/10.5281/zenodo. 1129431.

(67) Piggot, T. GROMOS-CKP POPS Simulations (versions 1 and 2) $298 \mathrm{~K}$ with GROMOS NH3 Charges and PME. 2017; https://doi.org/10.5281/zenodo.1129435.

(68) Jämbeck, J. P. M.; Lyubartsev, A. P. Implicit Inclusion of Atomic Polarization in Modeling of Partitioning Between Water and Lipid Bilayers. Phys. Chem. Chem. Phys. 2013, 15, 4677-4686.

(69) Piggot, T. Slipids POPS Simulations (versions 1 and 2) 298 K 1.0nmCut-off with LJ-PME. 2017; https://doi.org/10.5281/zenodo.1129441.

(70) Piggot, T. CHARMM36 DOPS Simulations (versions 1 and 2) 303 K 1.0nmLJ Switching. 2017; https://doi.org/10.5281/zenodo.1129411.

(71) Piggot, T. CHARMM36-Ua DOPS Simulations (versions 1 and 2) $303 \mathrm{~K}$ 1.0nmLJ Switching. 2017; https://doi.org/10.5281/zenodo.1129456.

(72) Kav, B.; Miettinen, M. S. Molecular Dynamics Simulation Trajectory of an Anionic Lipid Bilayer: 100 Mol\% DOPS with Na+ Counterions Using Joung-Cheetham Ions. 2018; https://doi.org/10.5281/zenodo.1134871. 
(73) Kav, B.; Miettinen, M. S. Molecular Dynamics Simulation Trajectory of an Anionic Lipid Bilayer: 100 Mol\% DOPS with Na+ Counterions Using Ff99 Ions. 2018; https : //doi.org/10.5281/zenodo.1135142.

(74) Piggot, T. Berger DOPS Simulations (versions 1 and 2) 303 K 1.0nmCut-Off. 2017; https://doi.org/10.5281/zenodo.1129419.

(75) Piggot, T. GROMOS-CKP DOPS Simulations (versions 1 and 2) $303 \mathrm{~K}$ with Berger/Chiu NH3 Charges and PME. 2017; https://doi.org/10.5281/zenodo. 1129429.

(76) Piggot, T. GROMOS-CKP DOPS Simulations (versions 1 and 2) $303 \mathrm{~K}$ with GROMOS NH3 Charges and PME. 2017; https://doi.org/10.5281/zenodo.1129447.

(77) Piggot, T. Slipids DOPS Simulations (versions 1 and 2) 303 K 1.0nmCut-off with LJ-PME. 2017; https://doi .org/10.5281/zenodo.1129439.

(78) Favela-Rosales, F. MD Simulation Trajectory of a Fully Hydrated DOPS Bilayer: SLIPIDS, Gromacs 5.0.4. 2017. 2017; https://doi.org/10.5281/zenodo. 495510.

(79) Smith, D. E.; Dang, L. X. Computer Simulations of NaCl Association in Polarizable Water. J. Chem. Phys 1994, 100, 3757-3766.

(80) Dang, L. X.; Schenter, G. K.; Glezakou, V.-A.; Fulton, J. L. Molecular Simulation Analysis and X-Ray Absorption Measurement of $\mathrm{Ca} 2+, \mathrm{K}+$ and $\mathrm{Cl}-$ Ions in Solution. J. Phys. Chem. B 2006, 110, 23644-54.

(81) Piggot, T. CHARMM36 POPS/POPC Simulations (versions 1 and 2) $298 \mathrm{~K} 1.0 \mathrm{~nm}$ LJ Switching with Na Ions. 2018; https://doi.org/10.5281/zenodo.1182665.

(82) Piggot, T. CHARMM36 POPS/POPC Simulations (versions 1 and 2) $298 \mathrm{~K} 1.0 \mathrm{~nm}$ LJ Switching with K Ions. 2018; https://doi.org/10.5281/zenodo.1182658. 
(83) Madsen, J. J. MD Simulations of Bilayers Containing PC/PS Mixtures and CaCl_2: 250POPC_50POPS_neutral. 2019; https://doi.org/10.5281/zenodo. 2542151.

(84) Madsen, J. J. MD Simulations of Bilayers Containing PC/PS Mixtures and CaCl_2: 250POPC_50POPS_0.15MCaCl_2. 2019; https://doi.org/10.5281/ zenodo. 2542176 .

(85) Madsen, J. J. MD Simulations of Bilayers Containing PC/PS Mixtures and CaCl_2: 250POPC_50POPS_1MCaCl_2.2019; https://doi.org/10.5281/zenodo. 2542135 .

(86) Nencini, R. PC/PS 5:1 Bilayer, Na Contraions, Additional CaC_2, CHARMM36 FF, NBFIX-Han. 2019; http://doi.org/10.5281/zenodo.3371327.

(87) Han, K.; Venable, R. M.; Bryant, A.-M.; Legacy, C. J.; Shen, R.; Li, H.; Roux, B.; Gericke, A.; Pastor, R. W. Graph-Theoretic Analysis of Monomethyl Phosphate Clustering in Ionic Solutions. The Journal of Physical Chemistry B 2018, 122, 14841494.

(88) Madsen, J. J. MD Simulations of Bilayers Containing PC/PS Mixtures and CaCl_2: 150POPC_150POPS_neutral. 2019; https://doi.org/10.5281/zenodo.2542164.

(89) Javanainen, M. Simulation of a POPC Bilayer at 298K, Lipid Model by Maciejewski and Rog. 2018; https://doi.org/10.5281/zenodo.1167532.

(90) Javanainen, M. Simulations of POPC/POPS Membranes with CaCl_2. 2017; https: //doi.org/10.5281/zenodo.1409551.

(91) Dickson, C. J.; Madej, B. D.; Skjevik, A. A.; Betz, R. M.; Teigen, K.; Gould, I. R.; Walker, R. C. Lipid14: The Amber Lipid Force Field. J. Chem. Theory Comput. 2014, $10,865-879$. 
(92) Kav, B.; Miettinen, M. S. Amber Lipid17 Simulations of POPC/POPS Membranes with KCl Counterions. 2018; https://doi .org/10.5281/zenodo.1250969.

(93) Kav, B.; Miettinen, M. S. Amber Lipid17 Simulations of POPC/POPS Membranes with NaCl Counterions. 2018; https://doi.org/10.5281/zenodo.1250975.

(94) Kav, B.; Miettinen, M. S. Amber Lipid17 Simulations of POPC/POPS Membranes with CaCl2. 2018; https://doi.org/10.5281/zenodo.1438848.

(95) Melcr, J. Molecular Dynamics Simulations of Lipid Bilayers Containing POPC and POPS with the Lipid17 Force Field, Only Counterions, and $\mathrm{CaCl} 2$ Concentrations. 2018; https://doi.org/10.5281/zenodo. 1487761.

(96) Tieleman, D. P.; Berendsen, H. J.; Sansom, M. S. an Alamethicin Channel in a Lipid Bilayer: Molecular Dynamics Simulations. Biophys. J. 1999, 76, 1757 - 1769.

(97) Ollila, O. H. S. POPC:POPS (4:1) Simulation with Berger Model at 310K. 2018; https://doi.org/10.5281/zenodo. 1475285.

(98) Cwiklik, L. MD Simulation Trajectory of a POPC/POPS (4:1) Bilayer with 102mM CaCl2, Berger Force Field for Lipids, Scaled Charges for Ca2+ and Cl-. 2017; https : //doi.org/10.5281/zenodo.887398.

(99) Cwiklik, L. MD Simulation Trajectory of a POPC/POPS (4:1) Bilayer with 715mM CaCl2, Berger Force Field for Lipids, Scaled Charges for Ca2+ and Cl-. 2017; https : //doi.org/10.5281/zenodo.887400.

(100) Piggot, T. GROMOS-CKP POPS/POPC Simulations (versions 1 and 2) 298 K with GROMOS NH3 Charges and PME. 2018; https://doi.org/10.5281/zenodo. 1283333.

(101) Piggot, T. GROMOS-CKP POPS/POPC Simulations (versions 1 and 2) $298 \mathrm{~K}$ 
with Berger/Chiu NH3 Charges and PME. 2018; https://doi.org/10.5281/zenodo. 1283331.

(102) Michaud-Agrawal, N.; Denning, E. J.; Woolf, T. B.; Beckstein, O. MDAnalysis: A Toolkit for the Analysis of Molecular Dynamics Simulations. J. Comput. Chem. 2011, 32, 2319-2327.

(103) Gowers, R. J.; Linke, M.; Barnoud, J.; Reddy, T. J. E.; Melo, M. N.; Seyler, S. L.; Domański, J.; Dotson, D. L.; Buchoux, S.; Kenney, I. M. et al. MDAnalysis: A Python Package for the Rapid Analysis of Molecular Dynamics Simulations. Proceedings of the 15th Python in Science Conference. 2016; pp 98 - 105.

(104) Abraham, M.; van der Spoel, D.; Lindahl, E.; Hess, B.; the GROMACS development team, GROMACS user manual version 5.0.7. 2015.

(105) Akutsu, H.; Seelig, J. Interaction of Metal Ions with Phosphatidylcholine Bilayer Membranes. Biochemistry 1981, 20, 7366-7373.

(106) Altenbach, C.; Seelig, J. Calcium Binding to Phosphatidylcholine Bilayers As Studied by Deuterium Magnetic Resonance. Evidence for the Formation of a Calcium Complex with Two Phospholipid Molecules. Biochemistry 1984, 23, 3913-3920.

(107) Seelig, J.; MacDonald, P. M.; Scherer, P. G. Phospholipid Head Groups As Sensors of Electric Charge in Membranes. Biochemistry 1987, 26, 7535-7541.

(108) Beschiaschvili, G.; Seelig, J. Peptide Binding to Lipid Membranes. Spectroscopic Studies on the Insertion of a Cyclic Somatostatin Analog into Phospholipid Bilayers. Biochimica et Biophysica Acta (BBA) - Biomembranes 1991, 1061, 78 - 84.

(109) Borle, F.; Seelig, J. Ca2+ Binding to Phosphatidylglycerol Bilayers As Studied by Differential Scanning Calorimetry and 2H- and 31P-Nuclear Magnetic Resonance. Chem. Phys. Lipids 1985, 36, 263 - 283. 
(110) Macdonald, P. M.; Seelig, J. Calcium Binding to Mixed PhosphatidylglycerolPhosphatidylcholine Bilayers As Studied by Deuterium Nuclear Magnetic Resonance. Biochemistry 1987, 26, 1231-1240.

(111) Roux, M.; Neumann, J.-M. Deuterium NMR Study of Head-Group Deuterated Phosphatidylserine in Pure and Binary Phospholipid Bilayers. FEBS Letters 1986, 199, 33-38.

(112) Melcr, J.; Martinez-Seara, H.; Nencini, R.; Kolafa, J.; Jungwirth, P.; Ollila, O. H. S. Accurate Binding of Sodium and Calcium to a POPC Bilayer by Effective Inclusion of Electronic Polarization. J. Phys. Chem. B 2018, 122, 4546-4557.

(113) Scherer, P. G.; Seelig, J. Electric Charge Effects on Phospholipid Headgroups. Phosphatidylcholine in Mixtures with Cationic and Anionic Amphiphiles. Biochemistry 1989, 28, 7720-7728.

(114) Roux, M.; Neumann, J.-M.; Bloom, M.; Devaux, P. F. 2H and 31P NMR Study of Pentalysine Interaction with Headgroup Deuterated Phosphatidylcholine and Phosphatidylserine. Eur. Biophys. J. 1988, 16, 267-273.

(115) Loosley-Millman, M.; Rand, R.; Parsegian, V. Effects of Monovalent Ion Binding and Screening on Measured Electrostatic Forces Between Charged Phospholipid Bilayers. Biophys. J. 1982, 40, $221-232$.

(116) Rand, R.; Parsegian, V. Hydration Forces Between Phospholipid Bilayers. Biochim. Biophys. Acta 1989, 988, $351-376$.

(117) Melcr, J. POPC Lipid Membrane, 303K, Charmm36 Force Field, Simulation Files and 200 Ns Trajectory for Gromacs MD Simulation Engine V5.1.2. 2016; https: //doi.org/10.5281/zenodo. 153944. 
(118) Petrache, H. I.; Tristram-Nagle, S.; Gawrisch, K.; Harries, D.; Parsegian, V. A.; Nagle, J. F. Structure and Fluctuations of Charged Phosphatidylserine Bilayers in the Absence of Salt. Biophys. J. 2004, 86, $1574-1586$.

(119) Ollila, S.; Hyvönen, M. T.; Vattulainen, I. Polyunsaturation in Lipid Membranes: Dynamic Properties and Lateral Pressure Profiles. J. Phys. Chem. B 2007, 111, $3139-3150$.

(120) Cevc, G. Membrane Electrostatics. Biochim. Biophys. Acta 1990, 1031, 311 - 382.

(121) Melcr, J.; Ferreira, T.; Jungwirth, P.; Ollila, O. H. S. Improved Cation Binding to Lipid Bilayer with Negatively Charged POPS by Effective Inclusion of Electronic Polarization. https://github.com/ohs0llila/ecc_lipids/blob/ master/Manuscript/manuscript.pdf, Submitted. 


\section{Graphical TOC Entry}

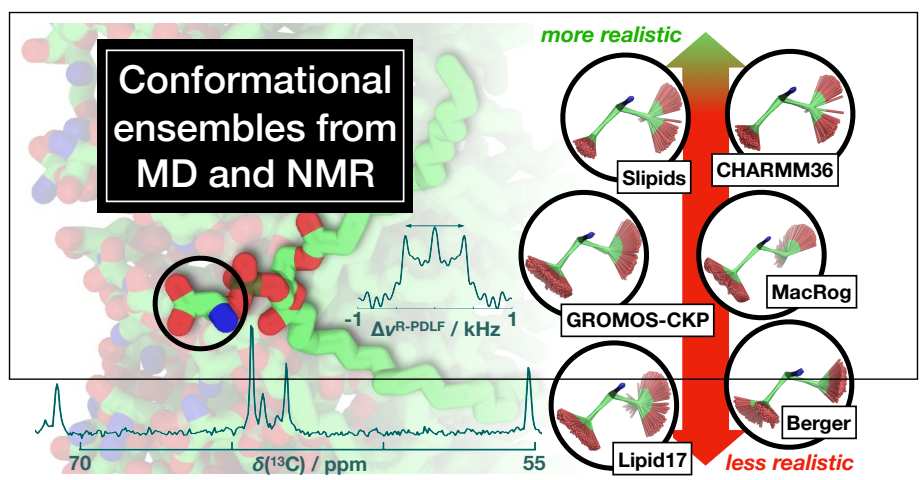

